\title{
DETECTION OF FORCHLORFENURON IN GRAPES BY SURFACE- ENHANCED RAMAN SPECTROSCOPY AND HPLC
}

\author{
A thesis \\ Presented to \\ The Faculty of the Graduate school \\ At the University of Missouri \\ In Partial Fulfillment \\ Of the Requirements for the Degree \\ Master of Science \\ By \\ HAOBING QIAN \\ Dr. Mengshi Lin, Thesis supervisor
}

December 2013 
The undersigned, appointed by the dean of the Graduate School,

have examined the thesis entitled

\title{
DETECTION OF FORCHLORFENURON IN GRAPES BY SURFACE-ENHANCED RAMAN SPECTROSCOPY AND HPLC
}

\author{
Presented by HAOBING QIAN
}

A candidate for the degree of Master of Science

And hereby certify that, in their opinion, it is worthy of acceptance.

Dr. Mengshi Lin, Food Science Department

Dr. Fu-hung Hsieh, Food Science Department

Dr. Liqun Gu, Biological Engineering Department 


\section{ACKNOWLEDGEMENTS}

I was admitted to the Graduate Program of Food Science Department at the University of Missouri (MU) in 2011. At the time of writing this thesis, it is my fifth and last semester here with my Master's study approaching completion. Now I can still remember the day when I received the news that I was admitted to MU, that was once-in-a-lifetime memory that I will never forget and I still feel so lucky to be able to live and study here for two and half years.

I would like to thank my advisor, Dr. Mengshi Lin who is always so kind and encouraging to me. Whenever I made a mistake or got stuck in the experiment, he never blamed me, always guided me through dark days, gave me useful advice, and helped me overcome all the difficulties in my research.

I want to express my appreciation to the help from my committee members, Dr. Fu-hung Hsieh and Dr. Liqun (Andrew) Gu. It is my luck and honor to have you on my committee. I'd like to thank you for your valuable suggestions about my study and research. In addition, you also taught me valuable knowledge in your course and helped me understand that to be an outstanding professional in Food Science, I should have a strong passion for research. I also want to thank Dr. Gu for his kind help to offer me a chance to do research in your lab, which will be a valuable part of my research.

I would also like to thank Dr. Mustapha not only for the knowledge I learnt from your class, but also for caring about students' feeling that I can feel deep in heart. Your 
intelligence, your insight view towards Food Microbiology, your elegance all made me so much in fond of you. You are always so warm and your laughter made me happy as well. I appreciate Dr. Bongkosh Vardhanabhuti's guidance and effort to brought me to MU, Dr. Ingolf U. Gruen and Dr. Andrew Clarke for their enlightening classes; Dr. Hao Li, for providing help supporting my research; Ms. JoAnn Lewis for her patient assistance whenever I had a question or needed help from her. Although she has retired now, I wish her a happy life after retirement.

I'd like to thank all of my lab mates and friends, Kangzi Ren, Xiaomo Mao, Zhengshan Wang, and Huan Yan. We accompanied each other and encouraged each other whenever we encountered difficulties. And we had a lot of fun together. I also thank the help from Xuesong Song, Zhong Zhang, and Dieu-Trang Nguyen-Ha. You taught me a lot about how to do research.

Last but not least, I would like to express my deepest love and thank to my parents and grandparents. Without your unconditional love and support, I would never be the one I am today. 


\section{TABLE OF CONTENTS}

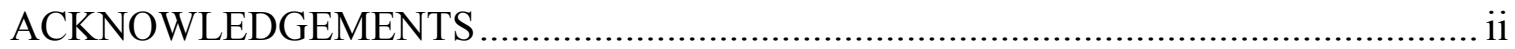

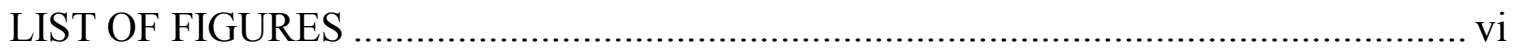

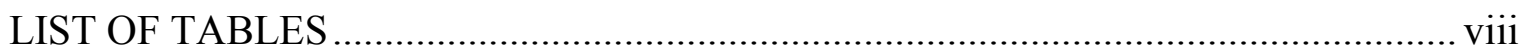

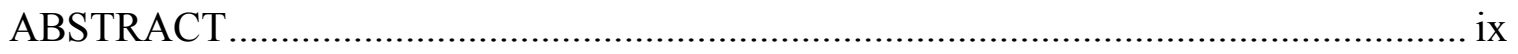

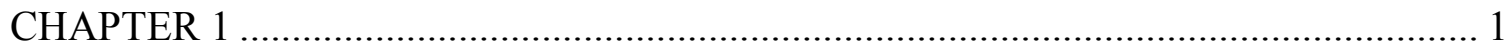

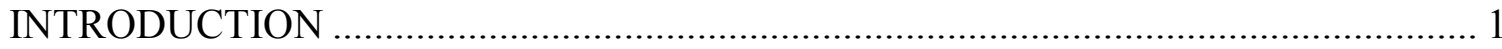

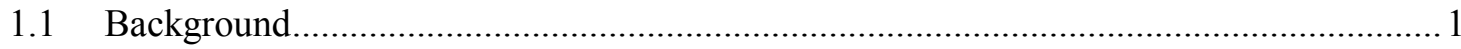

1.2 Objective

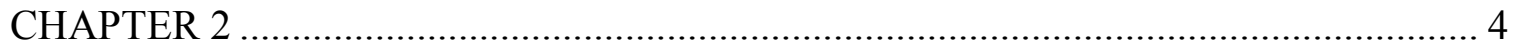

REVIEW OF LITERATURE ................................................................................. 4

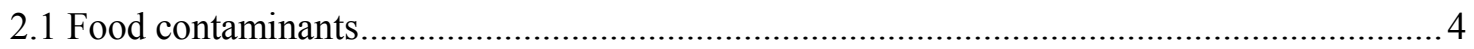

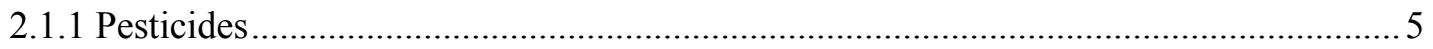

2.2 Conventional methods for detecting chemical contaminants ......................................... 8

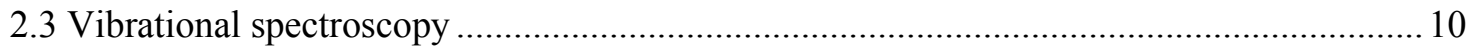

2.3.2 Surface-Enhance Raman Spectroscopy (SERS).................................................... 14

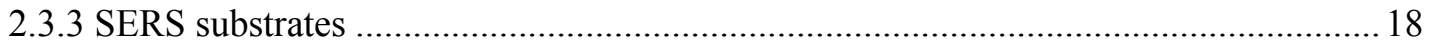

2.3.4 Applications of SERS in Food Science ..................................................................... 19

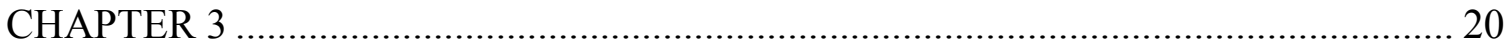

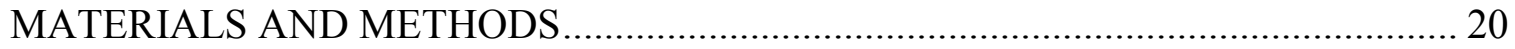

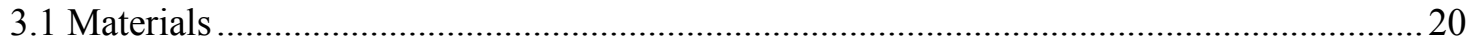

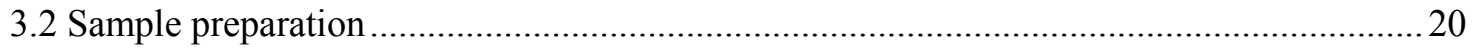

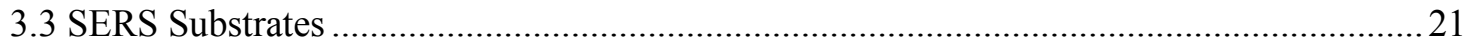

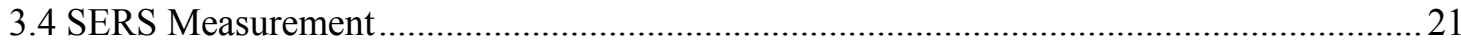

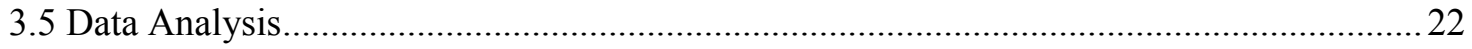

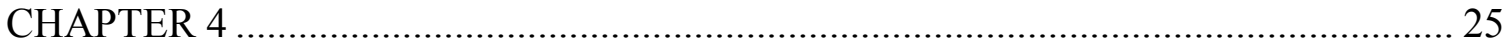




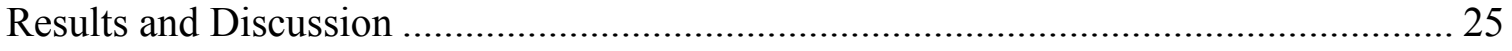

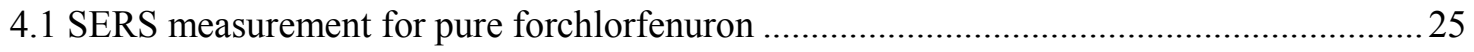

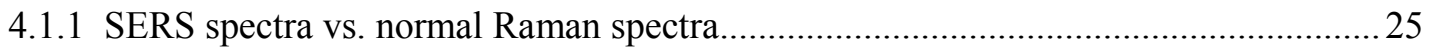

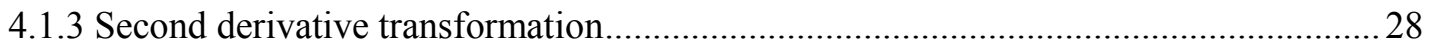

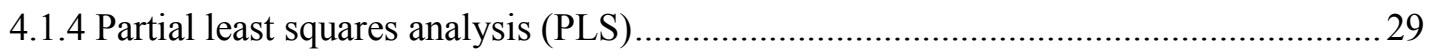

4.2 SERS measurement for forchlorfenuron extracted from grape skin................................. 32

4.2.1 Characteristic SERS spectra pattern for forchlorfenuron extraction at different

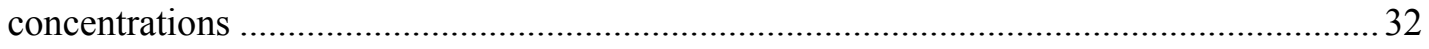

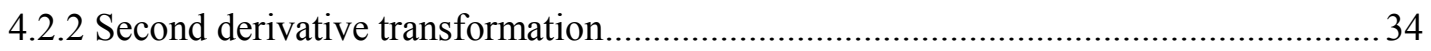

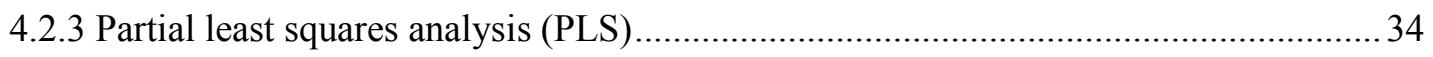

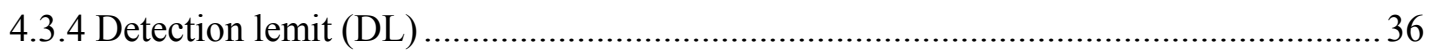

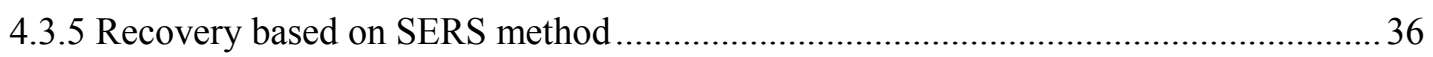

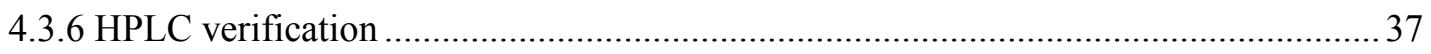

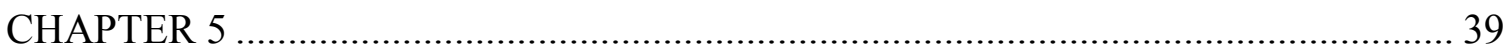

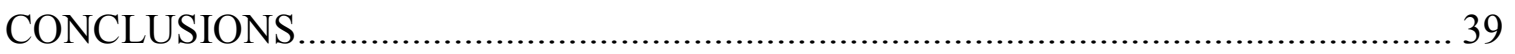

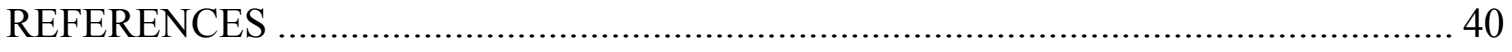

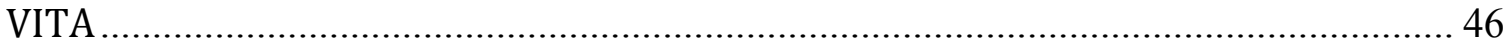




\section{LIST OF FIGURES}

Figure

Page

Figure 1. The outline of the experimental design. 3

Figure 2. Molecular structure of forchlorfenuron. 8

Figure 3. The major stages in an analytical procedure for measuring pesticide in fruit and vegetables.

Figure 4. Rayleigh and Raman scattering processes. 14

Figure 5. Illustration of the localized surface plasmon resonance effect (LSPR) (Tu and Chang 2012).

Figure 6. Normal Raman spectra and SERS spectra of $5 \mathrm{ppm}$ forchlorfenuron solution on gold slide and Q-SERS. Black line: forchlorfenuron solution tested on Q-SERS; Red line: forchlorfenuron solution tested on gold slide. Data were processed with smoothing at 4 $\mathrm{cm}^{-1}$ 26

Figure 7. Average SERS spectra $(n=5)$ of different concentrations of pure forchlorfenuron solutions. Spectra were processed by smoothing at $4 \mathrm{~cm}^{-1}$ and $2^{\text {nd }}$ polynomial subtraction for baseline adjustment.

Figure 8. Second derivative transformation of average SERS spectra $(n=5)$ at $1000 \mathrm{~cm}^{-1}$ for different concentrations of pure forchlorfenuron solutions.

Figure 9. RMSEP values of the partial least squares (PLS) models with different latent variables. 30

Figure 10. Predicted concentration (ppm) vs. actual concentration (ppm) of pure forchlorfenuron solutions using PLS models. Data processed with smoothing at $4 \mathrm{~cm}^{-1}$, $2^{\text {nd }}$ polynomial subtraction for baseline adjustment, four latent variables, spectral region from $600-1800 \mathrm{~cm}^{-1}$, spectral number $=40$.

Figure 11. Average SERS spectra $(n=7)$ of different concentrations of forchlorfenuron solutions extracted from grape skins. Spectra were processed by smoothing at $4 \mathrm{~cm}^{-1}$ and $2^{\text {nd }}$ order polynomial subtraction for baseline adjustment. 33

Figure 12. Second derivative transformation of average SERS spectra $(n=7)$ for different concentrations of forchlorfenuron solutions extracted from grape skins at $1000 \mathrm{~cm}^{-1} \ldots . .34$

Figure 13. RMSEP values of the PLS models with different latent variables. 35 
Figure 14. Predicted forchlorfenuron concentration (ppm) vs. actual puforchlorfenuron concentration (ppm) extracted from grape skin using PLS models at lowest latent variable. Data processed with smoothing at $4 \mathrm{~cm}^{-1}$, a $2^{\text {nd }}$ order polynomial subtraction for baseline adjustment, seven latent variables, spectral region from $600-1800 \mathrm{~cm}^{-1}$, spectral number $=35$. 35

Figure 15. Calibration curve for forchlorfenuron acquired by HPLC . .......................... 38 


\section{LIST OF TABLES}

Table

Page

Table 1. Band assignments of predominant peaks in SERS spectra of forchlorfenuron . 28

Table 2. Calculation of detection limits (DL) of SERS method for forchlorfenuron ....... 32

Table 3. Recovery of pure forchlorfenuron solutions .................................................. 37

Table 4. Recovery of forchlorfenuron solutions extracted from grape skins based on HPLC calibration curve 


\title{
DETECTION OF FORCHLORFENURON IN GRAPES BY SURFACE- ENHANCED RAMAN SPECTROSCOPY AND HPLC
}

\author{
Haobing Qian
}

Dr. Mengshi Lin, Thesis Supervisor

\begin{abstract}
The objective of this study was to use surface-enhanced Raman spectroscopy (SERS) for rapid detection and characterization of trace amounts of forchlorfenuron extracted from fruits. Forchlorfenuron is a plant growth regulator widely used in grapes. In this study, gold-coated nanosubstrates (Q-SERS ${ }^{\mathrm{TM}}$ ) were used for SERS measurements. Partial least squares (PLS) analysis was used as a statistical method for quantitative analysis of the spectral data. Our results demonstrate that enhanced Raman signals acquired from samples exhibited characteristic spectral patterns. The detection limit for forchlorfenuron by SERS is $3.14 \mathrm{ppm}$ at $99.86 \%$ confidence interval. The PLS results for quantification of forchlorfenuron were obtained: $\mathrm{R}=0.96, \mathrm{RMSEP}=9.336 \mathrm{ppm}$. In addition, HPLC was also used to measure forchlorfenuron and verify SERS results. A good linear relationship was observed between $0.1-100$ ppm with an R value of 0.999 at $260 \mathrm{~nm}$. These results demonstrate that SERS coupled with gold nanosubstrates is a rapid and simple method that requires little sample preparation. It could be a practical and
\end{abstract}


economical approach to combine SERS with HPLC to screen and analyze large number of food samples for detecting chemical contaminants and residues in fruits. 


\section{CHAPTER 1}

\section{INTRODUCTION}

\subsection{Background}

Forchlorfenuron or CPPU (1-[2-chloro-4-pyridyl]-3-phenyl urea) is a synthetic cytokinin, approved by the US Environmental Protection Agency (EPA) in 2004 as a plant growth regulator (EPA 2004). Forchlorfenuron is used in grapes, raisins, kiwifruits, and melons together with auxins synergistically to improve fruit size and weight.

Although there are few reports regarding the safety issue of forchlorfenuron except for its kidney toxicity based on a 2-year rat feeding study (EPA, 2004), excessive use of forchlorfenuron can cause exploding of fruits, contamination of the environment and negative long-term effects on human health.

Much attention has been drawn on monitoring the chemical contaminants in fruits and vegetables because most of them are eaten raw, which usually results in higher levels of chemical residues compared to other food groups (Chen and others 2011). Current methods to detect and quantify forchlorfenuron include high-performance liquid chromatography (HPLC) (Chen and others 2011; Hu and Li 2006), mass spectrometry (Valverde and others 2010), immunochromatographic assay (Suárez-Pantaleón and others 2012), and other chromatography-based methods. However, these methods are time-consuming, labor-intensive, and expensive. Therefore, novel analytical techniques 
are needed for rapid detection of chemical contaminants in foods with high accuracy and sensitivity.

Raman spectroscopy is one of the vibrational spectroscopic methods and has been considered a useful analytical technique to assess food quality ( $\mathrm{Lu}$ and others 2011). Inelastic light scattering of the incident light from a sample creates the Raman signals, which is related to characteristic molecular vibrations of analyte molecules. This gives unique Raman shift in the frequency or wavelength resulting in characteristic Raman emission (Kneipp and others 1999).

\subsection{Objective}

The overall goal of this project was to develop surface enhanced Raman spectroscopy (SERS) methods coupled with novel nanosubstrates and evaluate their performance in detection, characterization, and quantification of forchlorfenuron (CPPU) involved in fruits and vegetables especially from grapes for my project. HPLC was also used to verify and compare with SERS results. Partial least squares analysis (PLS) was used as a statistical method to analyze the SERS spectral data. 


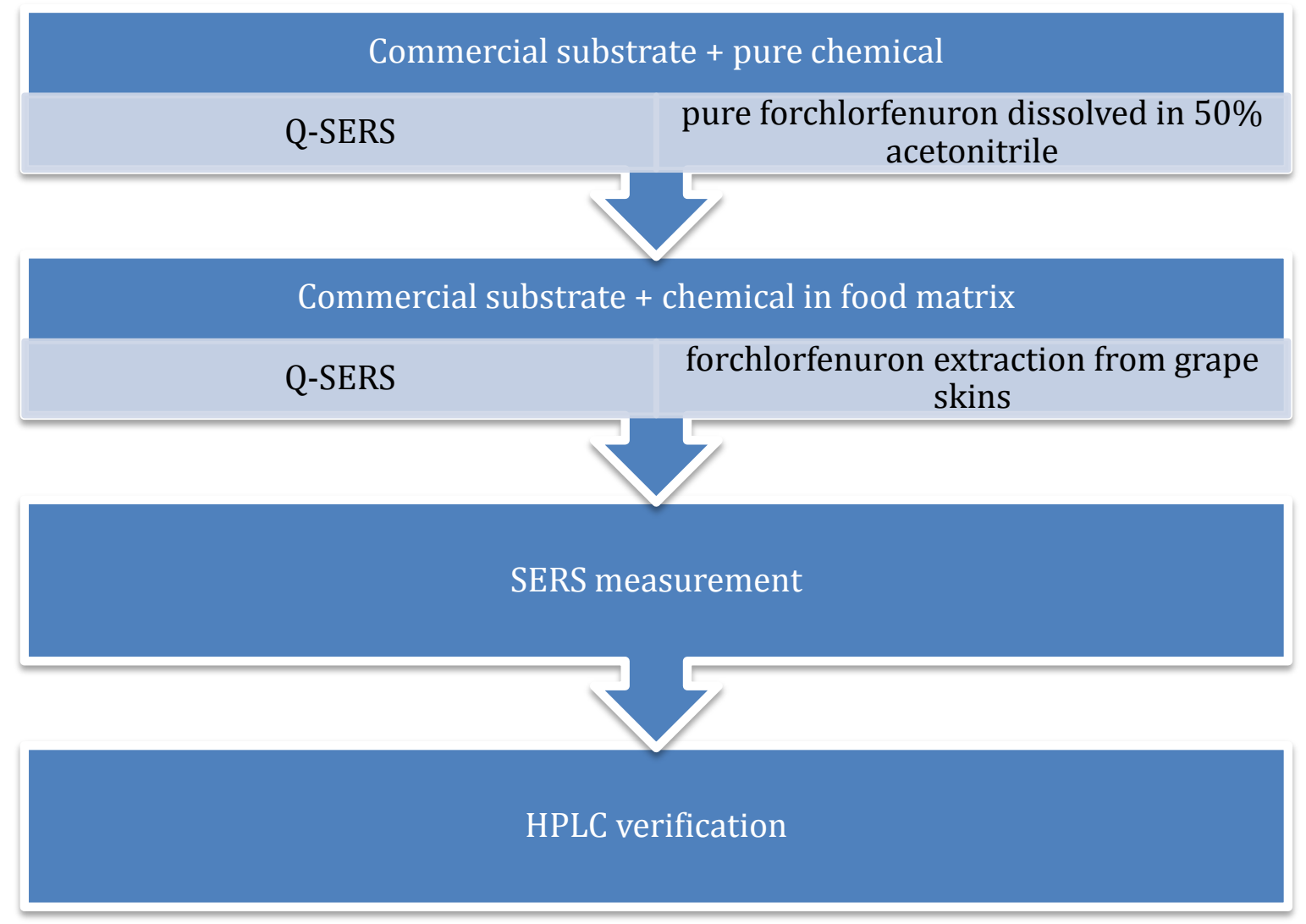

Figure 1. The outline of the experimental design. 


\section{CHAPTER 2}

\section{REVIEW OF LITERATURE}

\subsection{Food contaminants}

In recent years, there has been increasing concern about extensive use of pesticides, drugs, and other chemicals in foods such as fruits and vegetables, and processed foods. There are numerous types of chemical contaminants in food. They are widely used in various products for different purposes including increasing crop yields, ensuring the quality of crops and foods, and eliminating pests.

For example, contamination of melamine in pet foods and human foods caused a massive recall worldwide in 2007 and 2008 (Brown and others 2007; Cianciolo and others 2008), which led to a global scare for food safety issues (Chan and others 2008; Ingelfinger 2008; Xin and Stone 2008). Melamine is a nitrogen-rich chemical normally used in the production of dyes, fertilizers, fabrics, and other products. However, melamine has been intentionally added into pet foods, infant formula, and dairy products to boost the protein content. Melamine is a toxic compound to humans and animals. They are able to cause serious poisoning effects including chromic kidney inflammation, bladder carcinoma, and nephrolithiasis (Hau and others 2009). There is also a synergistic effect on toxicity when melamine is present with cyanuric acid, which may lead to renal failure in animals (Brown and others 2007; Puschner and others 2007).

Illegal dyes such as crystal violet (CV), fluoroquinolones (FQs), malachite green (MG) and other drug residues have been found in imported seafood from Asia and Mexico 
(FDA, 2007a, b). They are inexpensive anti-fungal and anti-parasite drugs used in aquaculture (Alderman 1988). However those chemicals have mutagenic and teratogenic effects on humans. Therefore they are banned for used in aquaculture in most countries (Culp and Beland 1996). Currently, there is a zero tolerance policy for these illegal dyes in fish as set by the FDA.

\subsubsection{Pesticides}

Pesticides are a major category of food contaminants. Pesticides are a diverse group of chemical compounds used to control pests in fruits and vegetables, and improve the quality and yields of crops and foods. They are widely used for the following purposes:

(1) Eliminate the pests that destroy the plant;

(2) Control microorganisms such as bacteria, molds, yeast, algae that cause spoilage in foods;

(3) Boost production yields;

(4) Stimulate or delay growing process for special needs.

However, they are one of the most toxic yet environmentally stable substances, thus posing a great risk to consumers (Cairns and Sherma 1992). These pesticide residues not only contaminate the crops on which they are applied, but also the environmental system including soil, water, and air. Pesticides can be divided into organic and inorganic compounds according to their structures. The organic pesticides include organophosphorous, organochlorine, and organonitrogen pesticides; while inorganic pesticides include fluoride insecticides, inorganic herbicides, inorganic fungicides, and 
arsenic insecticides. There are clear benefits of using pesticide in fruits and vegetables, but they also pose a great risk to human health because of their toxicity. Nowadays most countries have established regulations for the use of pesticides in agriculture. A common way is to set up a maximum residue level (MRL) for each kind of pesticides in different commodities. For example, in European Union (EU), the limit of detection (LOD) of several particular pesticides (acephate, aldrin, dichlorvos, fenthion) is $0.01-0.05 \mathrm{mg} / \mathrm{kg}$. The LOD of simazine is $0.1 \mathrm{mg} / \mathrm{kg}$ rising to $0.25 \mathrm{mg} / \mathrm{kg}$ in cherries. An LOD of $5 \mathrm{mg} / \mathrm{kg}$ is permissible for malathion in grapes and $7 \mathrm{mg} / \mathrm{kg}$ in tomatoes and tangerines (EU, 2013).

\subsubsection{Forchlorfenuron}

Forchlorfenuron or CPPU (1-[2-chloro-4-pyridyl]-3-phenyl urea) is a synthetic cytokinin, approved by the US Environmental Protection Agency (EPA) in 2004 as a plant growth regulator in the chemical class of Phenyl urea. Figure 2 shows the chemical structure of forchlorfenuron. Forchlorfenuron can be applied at early post-bloom by ground sprayer (EPA 2004). Plant growth regulators (PGRs) can grow endogenously by plants themselves or applied externally. They play a crucial role in regulating physiological processes in plant growth, such as cell division, differentiation, enlargement, ripening, germination, reproduction, and the protective responses against stress (Davies 1995; Han and others 2012). PGRs are used at different growth stages by applying foliar spray or bunch dipping to optimize berry growth and ensure the quality with desired size, shape, bunch, and weight. (Chadha and Shikhamany 1999). Forchlorfenuron is widely used in grapes, raisins, kiwifruits, and melons together with auxins (usually gibberellic acid 
(GA)). GA is a common phytohormone used in fruits and vegetables synergistically to improve fruit size and weight. It is often applied 48-50 days after fruit pruning according to "Good Agricultural Practices for Production of Quality Table Grapes" published by the National Research Center for grape in India. This is done either in dip application or slit dose along with GA or in foliar spray application (Sharma and Awasthi 2003). Although there are few reports regarding the safety issue of forchlorfenuron except for its kidney toxicity based on a 2-year rat feeding study (EPA, 2004), excessive use of forchlorfenuron can cause exploding of fruits, contamination of the environment and negative long-term effects on human health. Different organizations have issued the maximum residue levels for this chemical in different commodities. EPA has a regulation on the tolerance of forchlorfenuron residue in grapes and raisins with $0.06 \mathrm{ppm}$ (GPO, 2010). In EU, the residues of CPPU are regulated at the MRL of $0.01 \mathrm{ppm}$ (EFSA, 2012). Chinese government has set an MRL of $0.05 \mathrm{ppm}$ for forchlorfenuron in grapes (SHFDA, 2012). There has been increasing awareness in recent years about the residues of this synthetic chemical on fruits and vegetables because the FDA found that there is a growing trend of using pesticides and other chemicals on fruits from 2004 to 2007, as indicated by the fact that more domestic fruit samples were contaminated by residues of pesticides and other chemical substances (FDA 2007). 


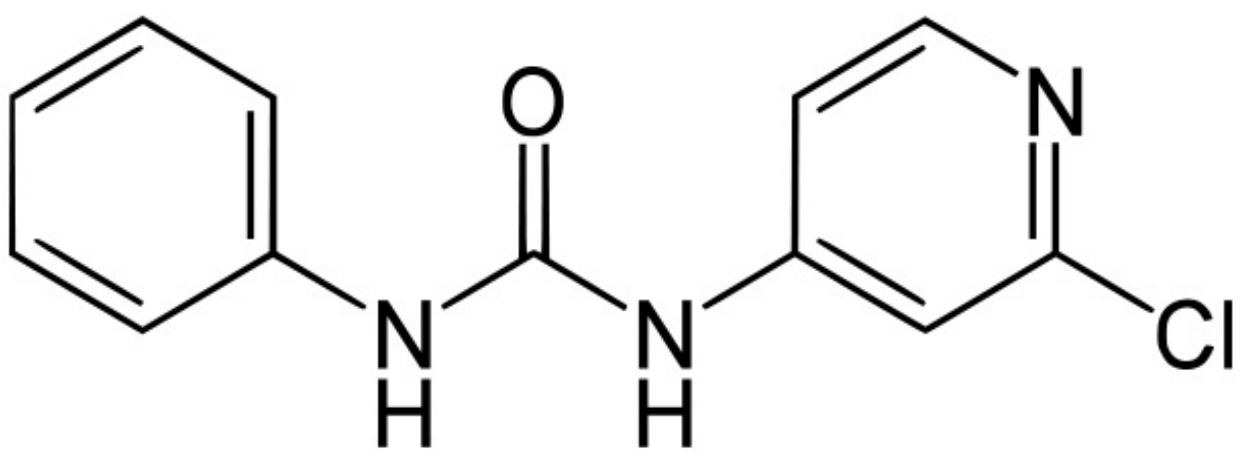

Figure 2. Molecular structure of forchlorfenuron.

\subsection{Conventional methods for detecting chemical contaminants}

Much attention has been drawn on monitoring the chemical contaminants in fruits and vegetables because most of them are eaten raw, which usually results in higher levels of chemical residues compared to other food groups (Chen and others 2011). There have been well-established traditional methods to detect and quantify forchlorfenuron, including high-performance liquid chromatography (HPLC) (Chen and others 2011; Hu and Li 2006), GC/MS (Valverde and others 2010), immunochromatographic assay (Suárez-Pantaleón and others 2012), and other chromatography-based methods. However, the procedure for detecting chemical contaminants in biological samples is a complex process and involves multiple steps, which are summarized in Figure 3 (Fenik and others 2011). 


\section{Sampling}

\section{Fixing, transportaton, and storage \\ Extraction, cleanup, and preparation for analysis \\ Qualitative and \\ quantitative analysis}

Figure 3. The major stages in an analytical procedure for measuring pesticide in fruit and vegetables.

Gas chromatography (GC) is one of the most commonly used analytical techniques for determining chemical contaminants in foods. GC can detect the residues of all classes of pesticide if equipped with a suitable column and detector. This technique is suitable for testing chemicals that are volatile and thermal stable. The type of chromatographic column is extremely important because it is the key part to separate analytes as well as to qualify and quantify the chemical. An ideal column should be highly efficient and resistant to changes in the separation process that is consists of two phases: solid phase and liquid phase. The solid phase should the thermal stable and highly selective to the constituents of the mixture being tested. If more than one pesticide are present in fruits and vegetables, a GC-MS is suitable for this situation due to its extremely efficient chromatographic separation capability, high sensitivity and confirmation power based on electron-impact ionization mass spectra (Fenik and others 2011). Compared to the 
conventional GC, GC-MS is more rapid and efficient. HPLC is another powerful technique for detecting and determining various chemical contaminants. It can be used especially when analytes are not suitable for determination by GC. These conventional techniques are well-established and have been widely used as standard methods by the FDA for detection of different types of chemicals in foods. For examples, LC-MS (Turnipseed and others 1998; Andersen and others 2006), HPLC/UV (FDA 2007), LC/MS/MS (Smoker and Krynitsky 2008), and GC/MS (Litzau and others 2008) have been used by the FDA for detection and identification of chemical contaminants and many illegal drugs in different food matrices. Generally, the LOD using chromatographybased methods could reach the ppb level.

However, these methods are time-consuming, labor-intensive, and expensive. Therefore, novel analytical techniques are needed for rapid detection of chemical contaminants in foods with high accuracy and sensitivity.

\subsection{Vibrational spectroscopy}

Vibrational spectroscopy is a spectroscopic technique based on molecular vibrations. The vibration occurs when the molecule has a constant rotational and translational motion pattern with atoms periodically moving inside. A molecular vibration is excited when the molecule absorbs a quantum of energy. While a fundamental vibration is excited when the energy is absorbed by the molecule when it is in the ground state. There are two categories of vibrational spectroscopy: one is infrared spectroscopy including Fourier transform infrared spectrometer (FTIR) and two-dimensional infrared correlation spectroscopy, the other is Raman spectroscopy. 


\subsubsection{Traditional Raman spectroscopy}

Raman spectroscopy is one of the vibrational spectroscopic methods and has been considered a useful analytical technique to assess food quality ( $\mathrm{Lu}$ and others 2011). Typically it uses a non-ionizing laser coupled with an incident beam as the excitation source. The incident photons from the laser source can be absorbed, scattered, and pass through the material without interaction. The photons are most likely to be absorbed and the molecule will be promoted from the ground state to the excited state if the energy gap of the molecule between these two states fits the energy of the incident photons (Tu and Chang 2012). The excited molecule then is able to be subsequently relaxed to the ground state by emission. There are two types of emission: stimulated and spontaneous emission. Stimulated emission is almost negligible because it can only occur when there is the incident photon energy and cannot occur after energy relaxation. While the spontaneous emission may occur at any energy level, which accordingly plays a major role in the emission process.

Fluorescence is the most common form that is one of the types of luminescence in order for us to observe the emission of a photon. It is a two-step process and can be described as follows (Le Ru and Etchegoin 2008):

1. An incident (laser) beam at lower level of energy $E_{L}$ excites an electron from ground state $\mathrm{S}_{0}$ to excited state $\mathrm{S}_{1}$. This is an absorption (dipole allowed) process.

2. The electron then relaxes down the vibrational sub-structure of $S_{1}$ through solvent interactions and others within 0.1-10 ps. As a result, it relaxes down to the lowest energy level of $S_{1}$. After that, several transitions may possibly happen. For example, spontaneous 
emission may occur with an average lifetime of $1-100 \mathrm{~ns}$, which corresponds to the electron relaxation down to a vibrational level in the sub-structure of the ground state $\mathrm{S}_{0}$. This emitted photon is associated with the fluorescence process.

Fluorescence occurs at the same time with spontaneous emission. The energies of the fluorescent photons differ from each other and the fluorescence intensity depends on the wavelength which gives the unique spectral pattern of different tested chemicals. The spectrum reflects the fingerprint-like profile of the underlying electronic and vibrational structure of the molecules, which is related to characteristic molecular vibrations of analyte molecules. This gives unique Raman shift in the frequency or wavelength resulting in characteristic Raman emission (Kneipp and others 1999). Therefore, Raman spectroscopy can provide useful vibrational spectral information on various chemicals and biochemical components.

On the other hand, when the electron clouds are distorted by the incident photon, the scattering may take place. There are typically two kinds of scatterings existing in the near-infrared and visible light spectral ranges: Raman and Rayleigh scattering. Rayleigh scattering is a more intense form than Raman scattering and only occur when the electron clouds are distorted. This is an elastic process and no energy exchange happens. Meanwhile if there exists energy transfer triggered by the alteration of the vibrational state of the molecule, Raman scattering takes place, which is an inelastic process. This Raman scattering is usually weak and only one out of $10^{6}-10^{8}$ photons undergoes the inelastic process (Mansour and Hickey 2007; Smith and Dent 2005; Gremlich and Yan 2001), which is indicated by a low Raman signal intensity. 
Based on the different direction of energy transfer between the photon and the molecules, Raman scattering can be further divided into two sub-categories: Stokes and anti-Stokes. Stokes scattering occurs when the molecule absorbs energy from the incident photon and promotes itself to a vibrational excited state from a lower energy level. On the contrary, anit-Stokes scattering may take place if the molecule has been already in the vibrational excited state before interacting with the incident photon because of prior external excitation or thermal disturbance. In this situation, due to the fact that scattered photon possesses a higher energy level than the incident photon, the scattered photon will interact with the incident photon by releasing energy and gradually return to a lower energy level. In most cases, as the molecules stay in the ground state at room temperature, stokes scattering therefore predominates the scattering pattern. The most commonly used unit describing the Raman band shifts, which refers to the energy difference between the scattered and incident photons, is wavenumber expressed in " $\mathrm{cm}^{-1}$ " and can be calculated through the following equation:

$\lambda=\mathrm{E} / \mathrm{hc}$

where $\lambda$ stands for the wavelength; E stands for the energy; $h$ is the Planck constant and $\mathrm{c}$ is the speed of light.

Figure 4 is a scheme illustrating the Rayleigh, Stokes and anti-Stoles scattering processes. 
Virtual states

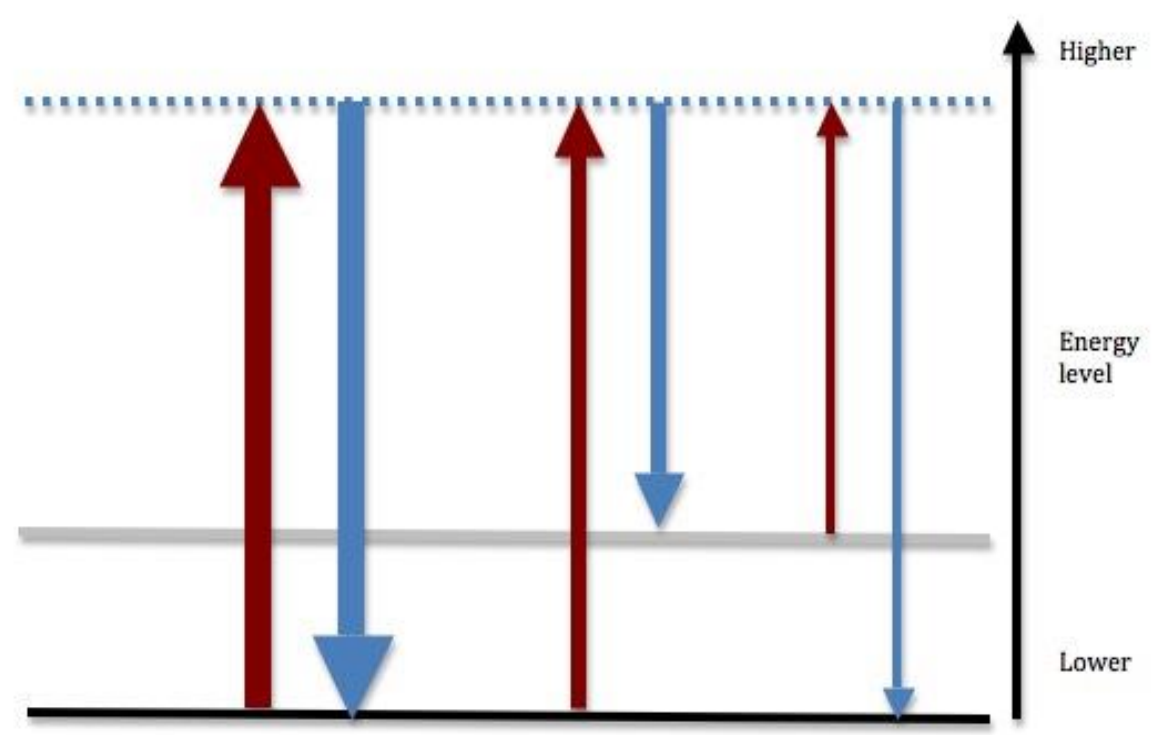

Figure 4. Rayleigh and Raman scattering processes.

One advantage of Raman spectroscopy is that chemicals can be tested in a complex system and is non-invasive. It requires minimum sample preparation with little destruction of samples (Naumann 2000; Li-Chan 1996). However, because only one out of one million photons undergoes Raman scattering, Raman spectroscopy is only suitable for measuring components that are in high concentration (Li-Chan 1996).

\subsubsection{Surface-Enhance Raman Spectroscopy (SERS)}

Since the 1970s, scientists have discovered and developed surface-enhanced Raman spectroscopy (SERS) that can detect samples based on significantly enhanced Raman signals compared to traditional Raman (Fleischmann and others 1974; Albrecht and Creighton 1977; Jeanmaire and Van Duyne 1977). The signal level is increased by the interaction between the molecule and a nanostructured metal surface. The signal thereby 
can be significantly augmented by $10^{3}-10^{14}$ fold. The enhancement factor (EF) varies depending on the definition of the EF (Le Ru and others 2007; Park and Kim 2010). The most commonly used definition for SERS EF can be calculated by the following equation:

$\mathrm{EF}=\frac{I_{S E R S} / N_{\text {Surf }}}{I_{R S} / N_{\text {Vol }}}$

Where $I_{S E R S}$ and $I_{R S}$ are the SERS and regular Raman intensity, respectively. $N_{V o l}$ is the average number of molecules in the scattering volume for regular Raman, and $N_{\text {Surf }}$ is the average number of adsorbed molecules in the scattering volume for SERS (McFarland and others 2005; Van Duyne 1979). For SERS in colloidal solutions, the analytical EF or apparent enhancement factor (AEFs) is calculated by

$\mathrm{EF}=\frac{I_{S E R S} / C_{R S}}{I_{R S} / C_{S E R S}}$

where $I_{S E R S}$ and $I_{R S}$ are the peak intensities in a SERS spectrum and a normal Raman spectrum, respectively. $\mathrm{C}_{\mathrm{SERS}}$ and $\mathrm{C}_{\mathrm{RS}}$ are the analyte concentrations in the SERS measurement and the normal Raman measurement, respectively.

Because of significantly enhanced Raman signals, SERS is suitable for detecting molecules in extremely low concentrations, which makes it attractive for detection of trace amounts of chemical residues in foods. 
This significant enhancement is mainly attributed to two contributions: electromagnetic field enhancement and chemical enhancement (Kneipp and others 2002; Haynes and others 2005a).

\subsubsection{Electromagnetic mechanism (EM)}

The electromagnetic field enhancement factor is caused by localized surface plasmon resonance that is excited by electromagnetic radiation and arises the coherent oscillation of the surface conduction electrons (Willets and Van Duyne 2007). When the light is incident on the nanostructured substrate, it drives the electron into collective oscillation and then generates large electromagnetic fields on the surface of the nanosubstrate (Haynes and others 2005b). If the surface is rough, the wave may excite localized surface plamon resonance (LSPR) on the resurface, resulting in enormous amplification of the electromagnetic fields near the surface.

The LSPR refers to the resonance with the frequency of incident light caused by the collective oscillation of valence electrons in a metal nanoparticle. The excitation of LSPR results in two consequences: scattering of the resonant electromagnetic radiation, and large enhancement of electromagnetic fields at the surface of the roughened transition metal. Figure 5 shows the illustration of LSPR (Haynes and others 2005b). 


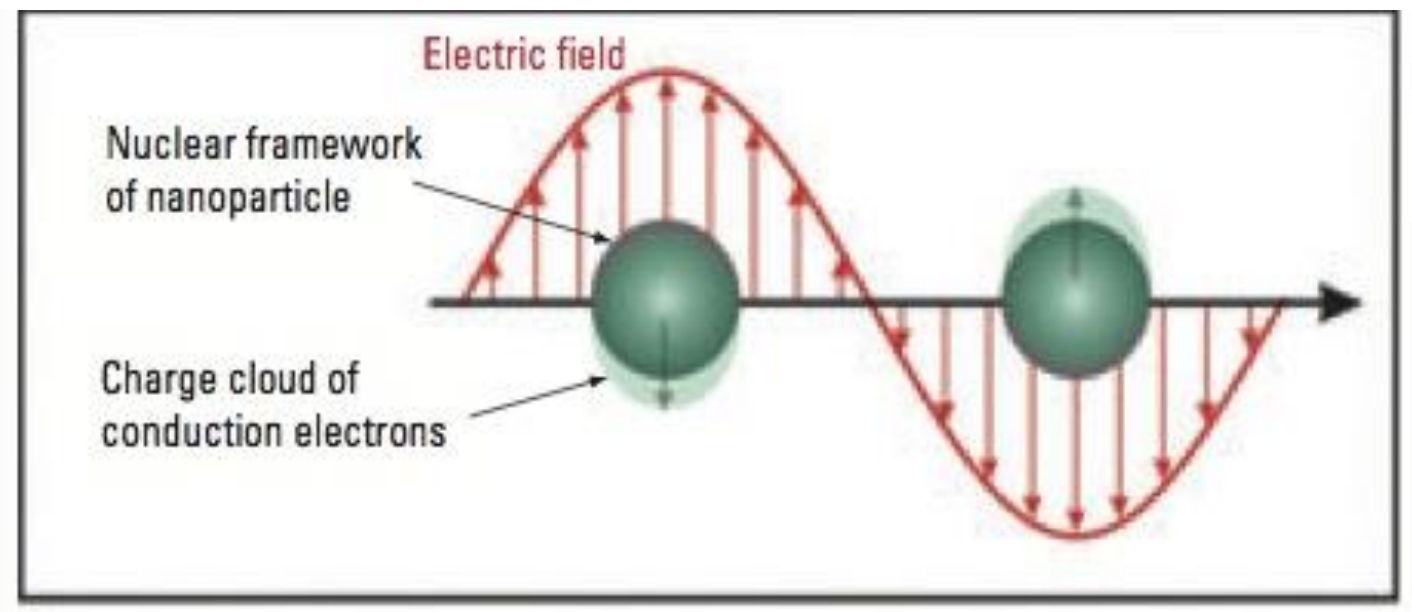

Figure 5. Illustration of the localized surface plasmon resonance effect (LSPR) (Tu and Chang 2012).

Theoretical modeling on the electromagnetic enhancement factor has been extensively established according to different fabrication of metal nanoparticles (Schatz 2001; Jensen and others 1999). The following one is a simplified model with an isolated sphere shaped nanoparticle:

$\mathrm{E}^{2} \propto \mathrm{E}_{0}^{2} \quad\left|\frac{\varepsilon_{m}-\varepsilon_{0}}{\varepsilon_{m}+2 \varepsilon_{0}}\right|^{2}$

in which $\mathrm{E}$ is the electric field magnitude at the surface of the sphere, $\mathrm{E}_{0}$ is the incident field magnitude, $\varepsilon_{\mathrm{m}}$ is the wavelength-dependent dielectric constant of the metal composing the sphere, and $\varepsilon_{0}$ is the dielectric constant of the local environment around the sphere (Schatz and Van Duyne 2002).

The electromagnetic enhancement plays a major role in SERS and has an average EF of $10^{4}$. The size, shape, and roughened surface of the nanomaterial are crucial factors that will alter this enhancement (Haynes and others 2005b). 


\subsubsection{Chemical enhancement mechanism}

Chemical enhancement mechanism has an average EF of 100 which is a charge-transfer state created between the metal and adsorbate molecules (Campion and Kambhampati 1998). When the molecule is adsorbed on the surface of metal particle, its magnitude, symmetry and resonant properties from the Raman polarizability of the isolated molecule will be altered. This plays a key role in systems where metal-to-molecule or molecule-tometal charge transfer occurs where the molecules are directly adsorbed to the roughened surface of the nanosubstrate, which drastically excites the resonances of the system and therefore contributing to so-called chemical enhancement (Moskovits 2005).

\subsubsection{SERS substrates}

Because SERS signal intensity is greatly influenced by the excitation of LSPR, choosing an appropriate or self-fabricating SERS metal substrates becomes the most important aspect in SERS analysis. There are many factors that can influence the LSPR, which then alter the signal intensity as well as ensure the reproducibility (Haynes and Van Duyne 2001). Factors such as shape, size, dielectric environment, and the inter-particle spacing of the material should be taken into consideration. An ideal SERS substrate can provide the desirable properties such as regularly or periodically arrangement of nanoparticles, reproducible fabrication of the substrate from array to array, homogenous signal enhancement across the surface, long-term stability, biocompatibility with biological chemicals tested like microorganisms, etc (Cialla and others 2012). Several types of SERS substrates have been developed. For example, metal island films are easy to fabricate and can provide a moderate EF between $10^{4}-10^{5}$. Colloidal nanoparticles are suitable for solution-phase SERS experiment. Various metallic nanoparticles are one of 
the major forms due to their low cost and easiness to fabricate. DNA-based nanoparticle assemblies and nanoparticle clusters are also known for providing hot spots. They are fabricated on the basis of single metallic nanoparticles with nanosized gaps. They have higher EF than single metallic ones (Lee and Irudayaraj 2009) because the intermolecular linkage by DNA or electrostatic interactions (Romo-Herrera and others 2011).

Nowadays, many novel nanosubstrates have been applied in SERS to increase Raman signals because they have a very high surface/volume aspect ratio, which ensures that a great number of probe molecules are captured in the close vicinity of the metal surface (Liu and others 2013a). Gold and silver are two most commonly used nanomaterials for use as SERS substrates (Kneipp and others 2002).

\subsubsection{Applications of SERS in Food Science}

There has been increasing use of SERS for rapid detection and characterization of different food contaminants, including melamine, cyanuric acid, organophosphophate, carbamate, and reactopamine (Lin and others 2008; Liu and others 2013b; Zhai and others 2011). The objective of this study was to extract forchlorfenuron from grape skins and to detect and characterize forchlorfenuron by SERS method coupled with gold nanosubstrates. Partial least squares analysis (PLS) was used as a statistical method to analyze the SERS spectral data. 


\section{CHAPTER 3}

\section{MATERIALS AND METHODS}

\subsection{Materials}

Forchlorfenuron was purchased from Sigma-Aldrich (St Louis, MO, USA). Organic grapes were purchased from a local market and washed to ensure no pesticide residues on grapes.

\subsection{Sample preparation}

A stock solution of forchlorfenuron $(100 \mu \mathrm{g} / \mathrm{mL})$ was prepared by dissolving forchlorfenuron powders in $50 \%$ acetonitrile that was used as the solvent system (acetonitrile : deionized water $=1: 1, \mathrm{v} / \mathrm{v}$ ), and stored at $4^{\circ} \mathrm{C}$. A serial concentration of forchlorfenuron solutions $(0,0.1,0.5,1,5,10,50$, and $100 \mathrm{ppm})$ were prepared by dilution from the stock solution. The $0 \mu \mathrm{g} / \mathrm{mL}$ solution was used as the control group.

The sample preparation procedure and extraction method were based on our previous paper (Liu and others 2013b) with some modifications. Briefly, grapes were weighed and their sizes were measured. The surface area of the fruit was then calculated accordingly. Based on the concentrations of standard forchlorfenuron solutions, the mass of forchlorfenuron that should be spiked on $1 \mathrm{~cm}^{2}$ of fruit skin was obtained. The samples were peeled and different concentrations of forchlorfenuron solutions were dropped evenly on $4 \mathrm{~cm}^{2}$ of grape skins. The skins of the samples were then blow-dried using an air dryer, cut into small pieces, and placed in conical tubes containing $4 \mathrm{~mL}$ of mixed solvent (acetonitrile/ $\mathrm{H}_{2} \mathrm{O}=1: 1, \mathrm{v} / \mathrm{v}$ ). The mixtures were vigorously vortexed for $1 \mathrm{~min}$ 
and then sonicated using an ultrasonic processor for $5 \mathrm{~min}$. The supernatants were filtered with a $0.45 \mu \mathrm{m}$ syringe filter. Finally, the filtrates were used for SERS measurement.

\subsection{SERS Substrates}

The SERS substrates, Q-SERS ${ }^{\mathrm{TM}} \mathrm{G}$, were obtained from Nanova Inc. (Columbia, MO, USA). Q-SERS ${ }^{\mathrm{TM}}$ substrates are gold-coated nanostructures fabricated on a silicon wafer. During the measurement, a volume of 0.3 to $0.5 \mu \mathrm{L}$ of the filtrate was dropped on the surface of a substrate using a micropipette. The substrate was then placed on a hot plate and heated at $35^{\circ} \mathrm{C}$ until the solvent completely evaporated.

\subsection{SERS Measurement}

A Renishaw RM1000 Raman spectrometer system (Gloucestershire, UK) equipped with a Leica DMLB microscope (Wetzlar, Germany) was used to test pure forchlorfenuron. This system is equipped with a $785 \mathrm{~nm}$ near-infrared diode laser source. During the measurement, light from the high power (maximum at $300 \mathrm{~mW}$ ) diode laser was directed and focused onto the sample at a microscope stage through a $\times 50$ objective. A $578 \times 385$ pixels charge-coupled device array detector detected Raman scattering signals. The size of each pixel was $22 \times 22 \mu \mathrm{m}$. Spectral data were collected by WiRE 3.2 software (Gloucestershire, UK). The detection range for forchlorfenuron is from 500 to $1800 \mathrm{~cm}^{-1}$. After measurement optimization, spectra of samples were collected using a $\times 50$ objective with $10 \mathrm{~s}$ exposure time, and $300 \mathrm{~mW}$ laser power. For forchlorfenuron extracted from the grape skin, the SERS measurement was conducted using a Renishaw InVia Raman 
microscope equipped with a Leica Microsystem CMC GmbH using $785 \mathrm{~nm}$ near-infrared diode laser source. The extracted samples were tested under the condition of $10 \mathrm{~s}$ exposure time, 1 accumulation, and a $\times 50$ objective with $5 \%$ laser power. The spectra were collected by Wire 3.3 software (Gloucestershire, UK).

For HPLC test, both pure and extracted samples were pretreated by centrifugation at $10000 \mathrm{rpm}$ at $4^{\circ} \mathrm{C}$ for $10 \mathrm{~min}$, and then stored at $4^{\circ} \mathrm{C}$ before testing. The measurement was conducted using Agilent 1100 system with a $\mathrm{C}_{18}$ column. Parameters for measurement included: a mobile phase of methanol/water $(63: 37 \mathrm{v} / \mathrm{v})$, temperature at $40^{\circ} \mathrm{C}, 260 \mathrm{~nm}$ detecting UV light, $1 \mathrm{~mL} / \mathrm{min}$ flow rate, and $10 \mu \mathrm{L}$ injection volume.

\subsection{Data Analysis}

SERS spectral data were collected and analyzed by Delight software version 3.2.1 (DSquared Development Inc., LaGrande, OR, USA). Data were first processed by second order polynomial subtraction to offset baseline shift. Gaussian smoothing was used at 4

$\mathrm{cm}^{-1}$ to eliminate high frequency instrumental noises. PLS linear regression analysis models were established to predict pesticide concentrations in the samples. The higher an $R$ value is obtained, the better predictability the model indicates (Huang and others 2002). The lowest root mean square error of prediction (RMSEP) value was used to choose the optimum number of PLS latent variables. RMSEP can be calculated in the following equation: 


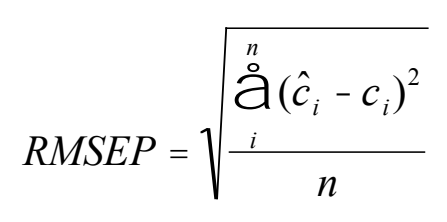

$C_{i}{ }^{\prime}$ is the predicted concentration (ppm) of forchlorfenuron solutions, $C_{i}$ is the actual concentration (ppm) of forchlorfenuron. A higher R value or a lower RMSEP value suggests a better predictability of the PLS model.

The detection limit (DL) for forchlorfenuron can be calculated and expressed from the PLS calibration curve according to both the International Union of Pure and Applied Chemistry (IUPAC) and the American Chemical Society (ACS).

$\mathrm{DL}=3 \delta / \mathrm{m}$

Where $\delta$ is the standard error in the y-intercept, $m$ is the slope of the PLS model, and 3 represents the model was conducted at $99 \%$ confidence interval.

The standard PLS calibration curve of forchlorfenuron was obtained by testing different concentrations of standard forchlorfenuron solutions. Two concentrations (5 and $50 \mathrm{ppm})$ of forchlorfenuron solutions extracted from the grape skins were selected to determine the recovery percentage, which can be calculated based on the following equation: 
Recovery $\%=\left(\mathrm{C}_{\text {quantified }}-\mathrm{C}_{\text {control }}\right) / \mathrm{C}_{\text {spiked }} \times 100 \%$

where $\mathrm{C}_{\text {quantified }}$ is the quantified forchlorfenuron concentration in the spiked grape samples, $\mathrm{C}_{\text {control }}$ is the forchlorfenuron concentration in the control grape samples, and $\mathrm{C}_{\text {spiked }}$ is the known concentration of spiked forchlorfenuron calculated according to the spiked amount of the pure forchlorfenuron solution and the weight of grape skins. 


\section{CHAPTER 4}

\section{Results and Discussion}

\subsection{SERS measurement for pure forchlorfenuron}

4.1.1 SERS spectra vs. normal Raman spectra

Both SERS and normal Raman spectra of 5 ppm forchlorfenuron solution were acquired using Q-SERS substrates and gold slides. As shown in Figure 6, the Raman signals of forchlorfenuron were significantly enhanced on Q-SERS substrates compared with normal Raman spectra. During measurement, a higher power of laser source may provide a better signal-to-noise ratio. However attention was paid to avoid oversaturation that may cause damage to the samples. 


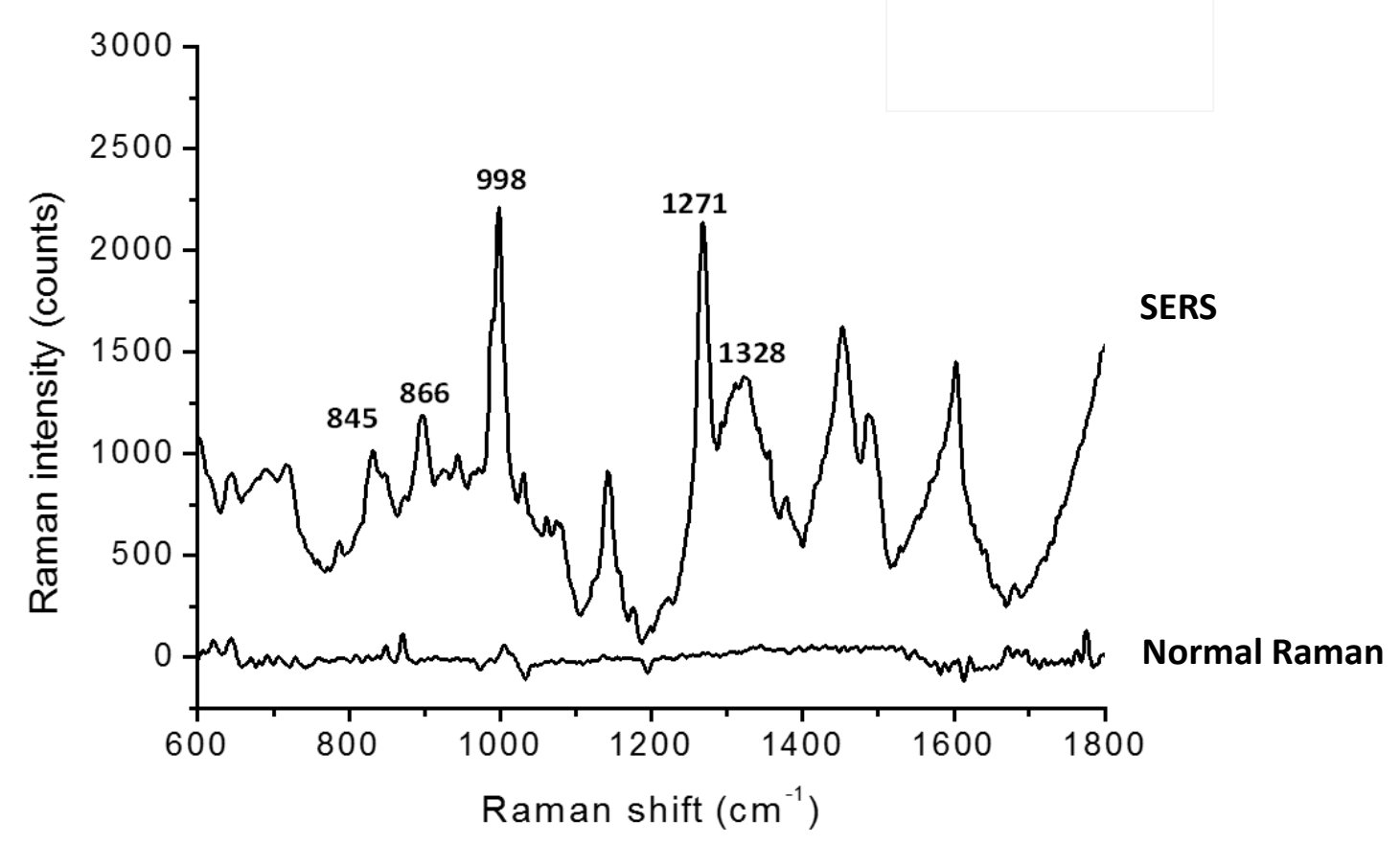

Figure 6. Normal Raman spectra and SERS spectra of 5 ppm forchlorfenuron solution on gold slide and QSERS. Black line: forchlorfenuron solution tested on Q-SERS; Red line: forchlorfenuron solution tested on gold slide. Data were processed with smoothing at $4 \mathrm{~cm}^{-1}$.

4.1.2 Characteristic SERS spectra pattern for forchlorfenuron at different concentrations

SERS spectra of different concentrations of pure forchlorfenuron solutions were obtained on Q-SERS. Average SERS spectra $(\mathrm{n}=5)$ of pure forchlorfenuron are shown in Figure 7. This result is consistent with the spectra of the powder form measured on Q-SERS, indicating that there is little interference from the organic solvent. Band assignments were summarized in Table 1. The most predominant peak of forchlorfenuron is at 998 $\mathrm{cm}^{-1}$ due to the symmetric ring stretching of monosubstituted benzene part together with the ring breathing of pyridine part. Other typical peak at $845 \mathrm{~cm}^{-1}$ can be attributed to 
substituent sensitive vibrations of the pyridine part here referring to the $\mathrm{C}$ - $\mathrm{Cl}$ linkage; $1271 \mathrm{~cm}^{-1}$ due to the $\mathrm{C}-\mathrm{N}$ asymmetric stretching vibration of monosubstituted benzene; $1328 \mathrm{~cm}^{-1}$ due to benzene ring skeletal vibration. As shown in Figure 3, the SERS spectra of forchlorfenuron were distinct compared to the control (acetonitrile solvent system). The Raman intensity of the predominant peaks increased with the increase of sample concentrations.

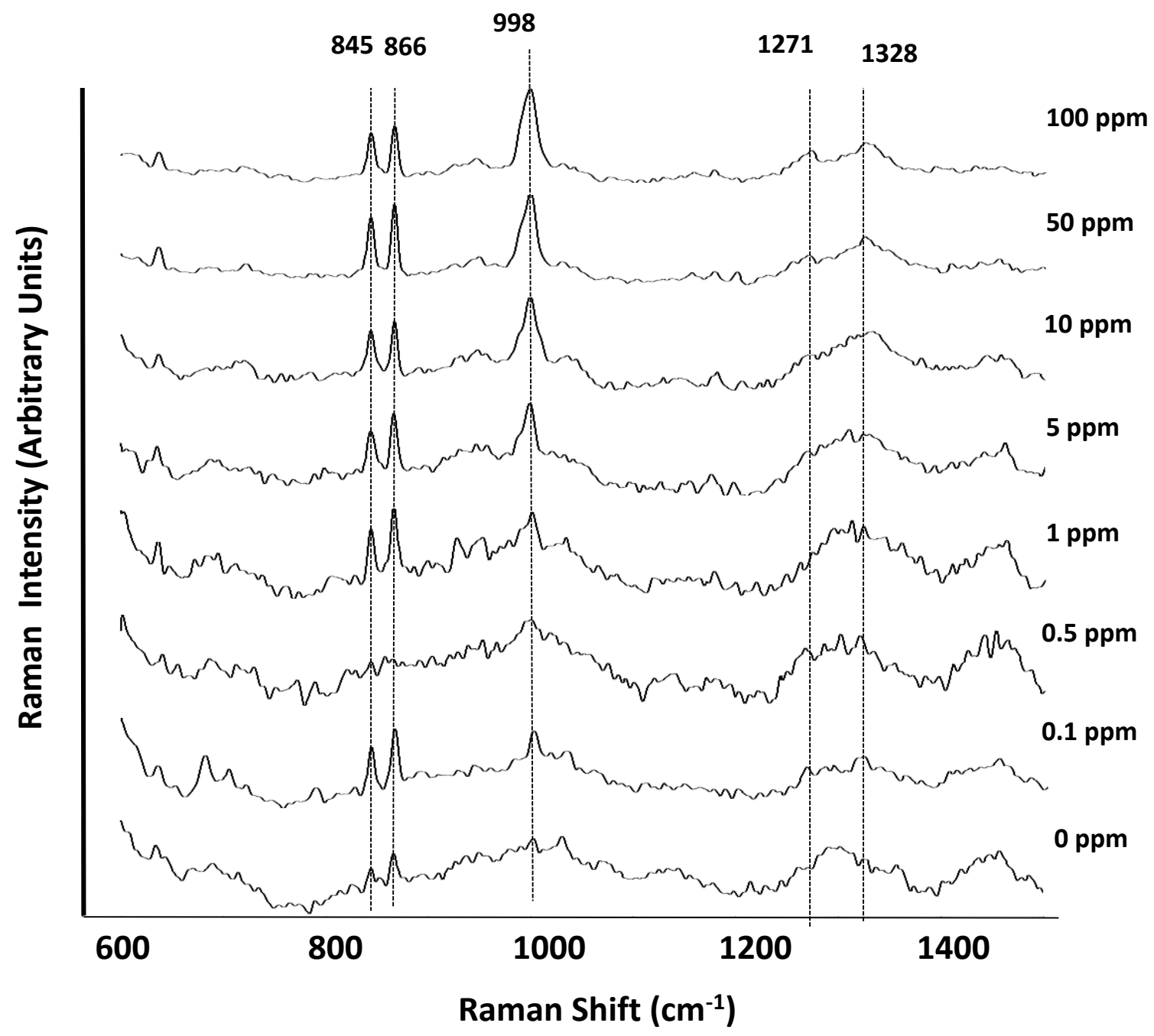

Figure 7. Average SERS spectra $(n=5)$ of different concentrations of pure forchlorfenuron solutions.

Spectra were processed by smoothing at $4 \mathrm{~cm}^{-1}$ and a $2^{\text {nd }}$ polynomial subtraction for baseline adjustment. 
Table 1. Band assignments of predominant peaks in SERS spectra of forchlorfenuron

Band $\left(\mathrm{cm}^{-1}\right) \quad$ Assignment

Monosubstituted benzene part

$615 \mathrm{w}$

$998 \mathrm{~s}$

$1028 \mathrm{~m}$

$1271 \mathrm{~m}$

$1328 \mathrm{~m}$

$1456 \mathrm{w}$

$1603 \mathrm{~m}$

Pyridine part (2-substitued)

$845 \mathrm{~m}$

$998 \mathrm{~s}$

$700 \mathrm{w}$

$637 \mathrm{w}$
Depolarized in-plane ring deformation

Symmetric ring stretch

In-plane $\mathrm{CH}$ stretching

C-N asymmetric stretching vibration

Ring skeletal vibration

Ring deformation vibration

Ring stretching vibration

Substituent sensitive vibration

Ring breathing

C-Cl stretching

NCO deformation vibrations

$w$ weak, $m$ medium, $s$ strong

(Freeman and Lord 1974; Jehlicka and others 2010; Dollish and others 1974; Klots 1995; Klots and Collier 1995)

\subsubsection{Second derivative transformation}

Figure 8 shows the second derivative transformation of the most predominant peak at 998 $\mathrm{cm}^{-1}$. Second derivative transformation was used to adjust baseline shifts and separate overlapping bands. This figure demonstrates that it can clearly differentiate between different concentrations of forchlorfenuron ranging from 5 to $100 \mathrm{ppm}$. However, for 
those spectral samples with concentrations of less than $5 \mathrm{ppm}$, it is difficult to differentiate them based on spectral features.

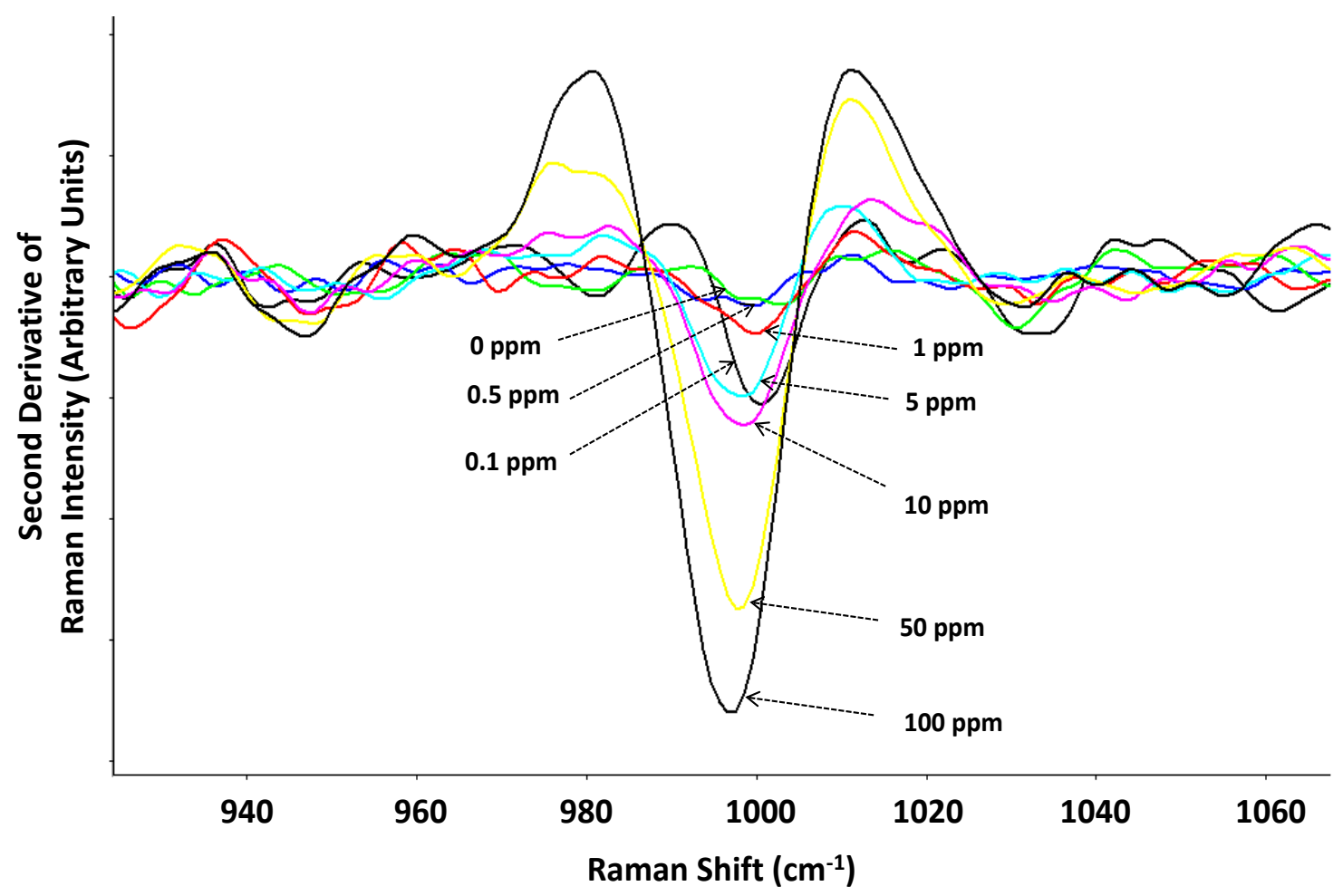

Figure 8. Second derivative transformation of average SERS spectra $(\mathrm{n}=5)$ at $1000 \mathrm{~cm}^{-1}$ for different concentrations of pure forchlorfenuron solutions.

4.1.4 Partial least squares analysis (PLS) 
RMSEP values obtained from the PLS model are shown in Figure 9. The lowest RMSEP value was achieved when applying four latent variables, indicating that the optimum RESEP value to construct the PLS model is four. A PLS model was established by plotting predicted concentration verse actual concentration of samples using four latent variables (Figure 10). R value is 0.96 and RMSEP is $0.9314 \times 10^{-5}$, suggesting that PLS is a reliable analytical tool for quantification of forchlorfenuron in solution.

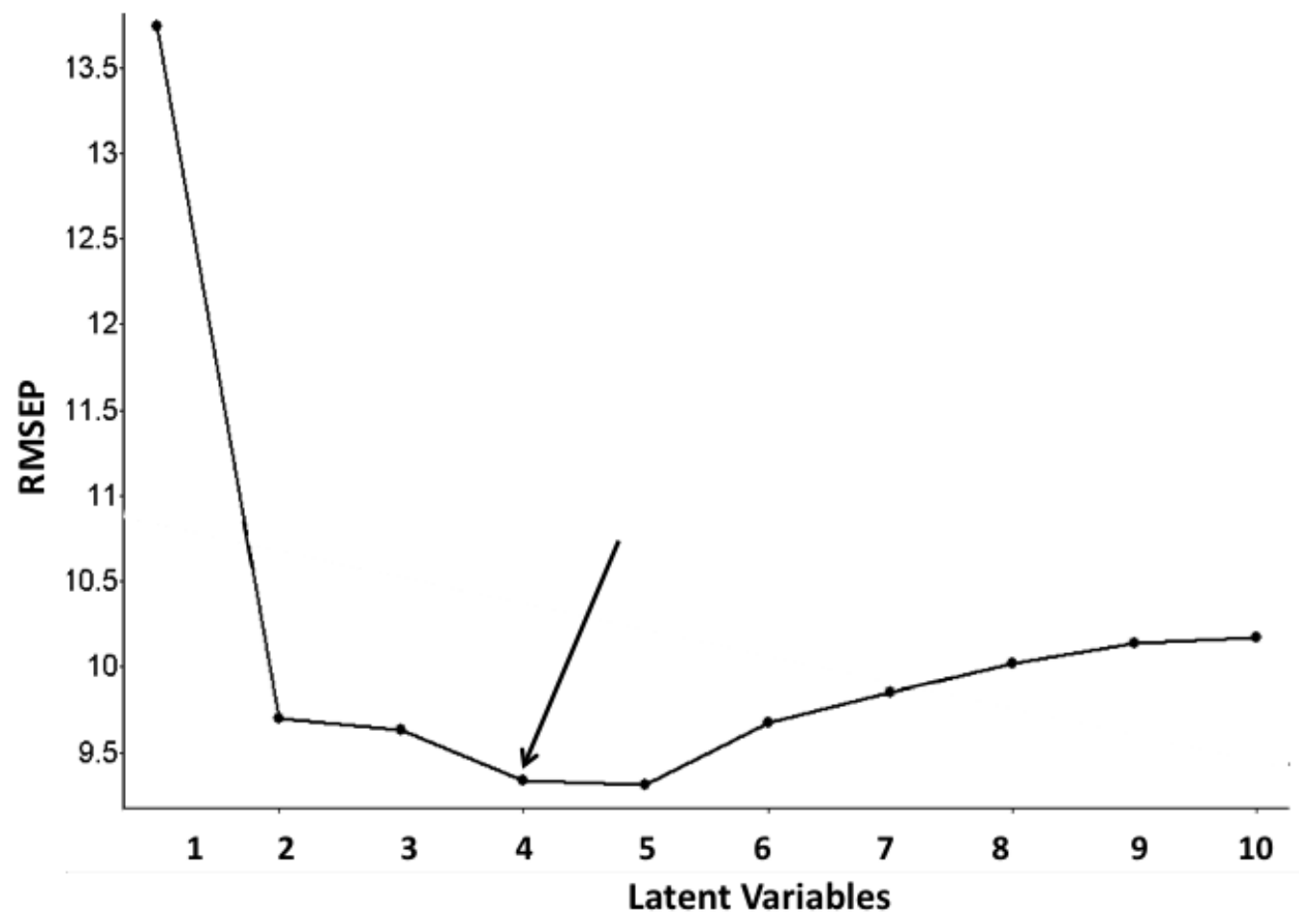

Figure 9. RMSEP values of the partial least squares (PLS) models with different latent variables. 


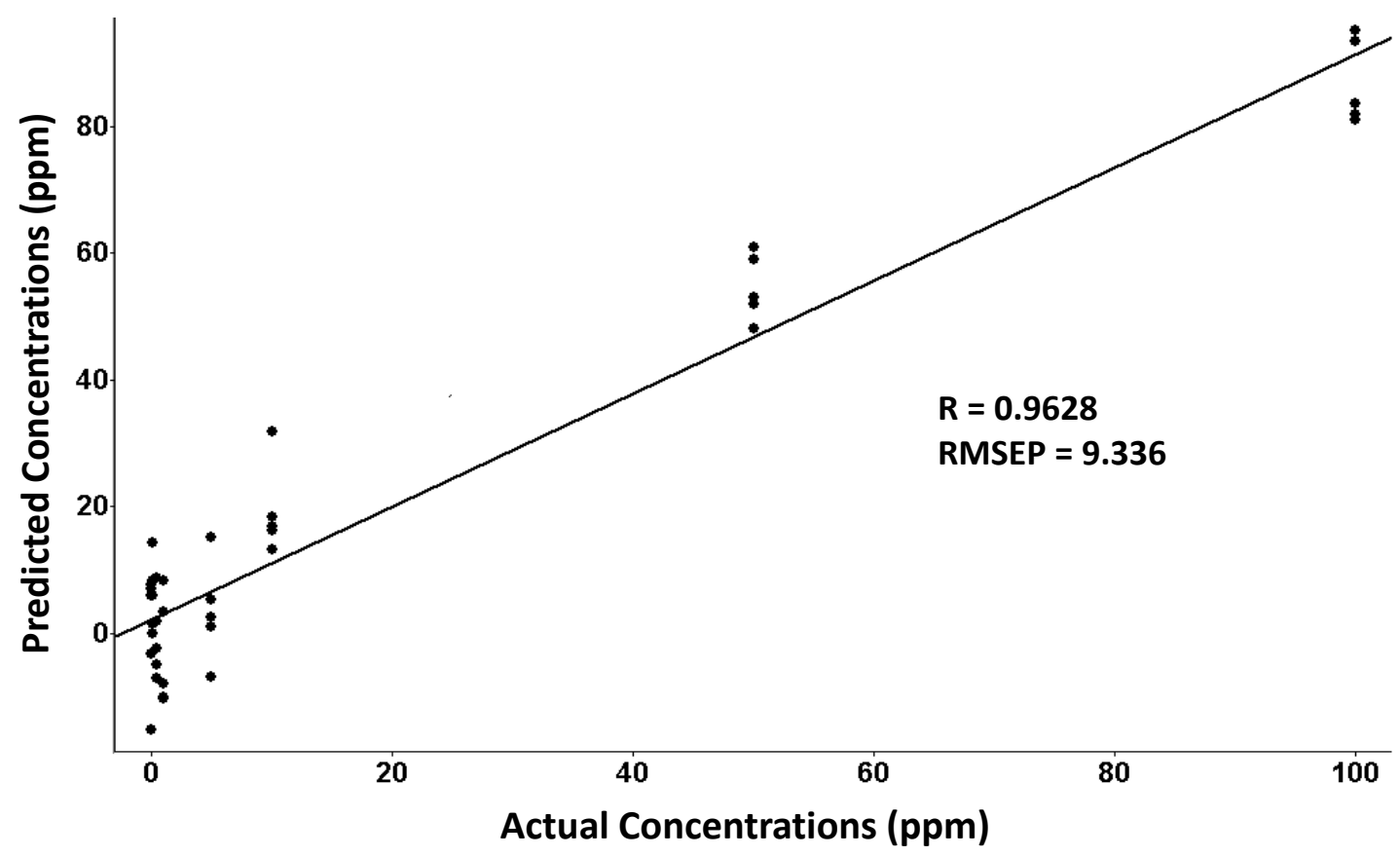

Figure 10. Predicted concentration ( $\mathrm{ppm}$ ) vs. actual concentration ( $\mathrm{ppm}$ ) of pure forchlorfenuron solutions using PLS models. Data processed with smoothing at $4 \mathrm{~cm}^{-1}, 2^{\text {nd }}$ polynomial subtraction for baseline adjustment, four latent variables, spectral region from $600-1800 \mathrm{~cm}^{-1}$, spectral number $=40$.

\subsubsection{Detection limit (DL)}

The DL of forchlorfenuron by SERS was calculated based on the equation 6 and are shown in Table 2. The DL for detection of pure forchlorfenuron solutions was $3.15 \mathrm{ppm}$ and $4.43 \mathrm{ppm}$ for samples extracted from the grape skin. This is in agreement with Figure 8 as concentrations fall below $5 \mathrm{ppm}$, the spectra are indistinguishable from each other. Next, the HPLC method was used to in this study further verify these results. 
Table 2. Calculation of detection limits (DL) of SERS method for forchlorfenuron

\begin{tabular}{lllll}
\hline Sample & $\mathrm{R}$ & Standard error & Slope & DL (ppm) \\
\hline $\begin{array}{l}\text { Pure } \\
\begin{array}{l}\text { Forchlorfenuron } \\
\text { Forchlorfenuron } \\
\text { extracted from } \\
\text { grape skin }\end{array}\end{array} 0.96$ & 0.93 & 0.886 & 3.15 \\
\hline
\end{tabular}

To predict the concentration of forchlorfenuron residues and calculate the recovery values, a calibration curve was established (Figure 11). Unlike traditional chromatographic methods of obtaining the calibration curve from pure samples first, and then quantifying the concentrations extracted from food samples accordingly, this method is based on the PLS models and predicts the concentrations of forchlorfenuron directly. As shown in Table 3, the recovery percentage of pure forchlorfenuron with concentrations of 50 and $100 \mathrm{ppm}$ are $111.97 \%$ and $79.04 \%$, respectively. While for lower concentrations of samples, the recovery are low (data not shown), suggesting that a better extraction method is needed to establish a more sensitive detection protocol with lower DL.

\subsection{SERS measurement for forchlorfenuron extracted from grape skin}

4.2.1 Characteristic SERS spectra pattern for forchlorfenuron extraction at different concentrations 
We also detected and quantified forchlorfenuron extracted from real fruit sample. Average spectra $(\mathrm{n}=7)$ of forchlorfenuron in five different concentrations ( 1 to $100 \mathrm{ppm})$ extracted from grape skins were acquired on Q-SERS (Figure 11). Spectral data were preprocessed by the same transformations as the pure samples. The spectral pattern was highly consistent with that from pure forchlorfenuron (Figure 7).

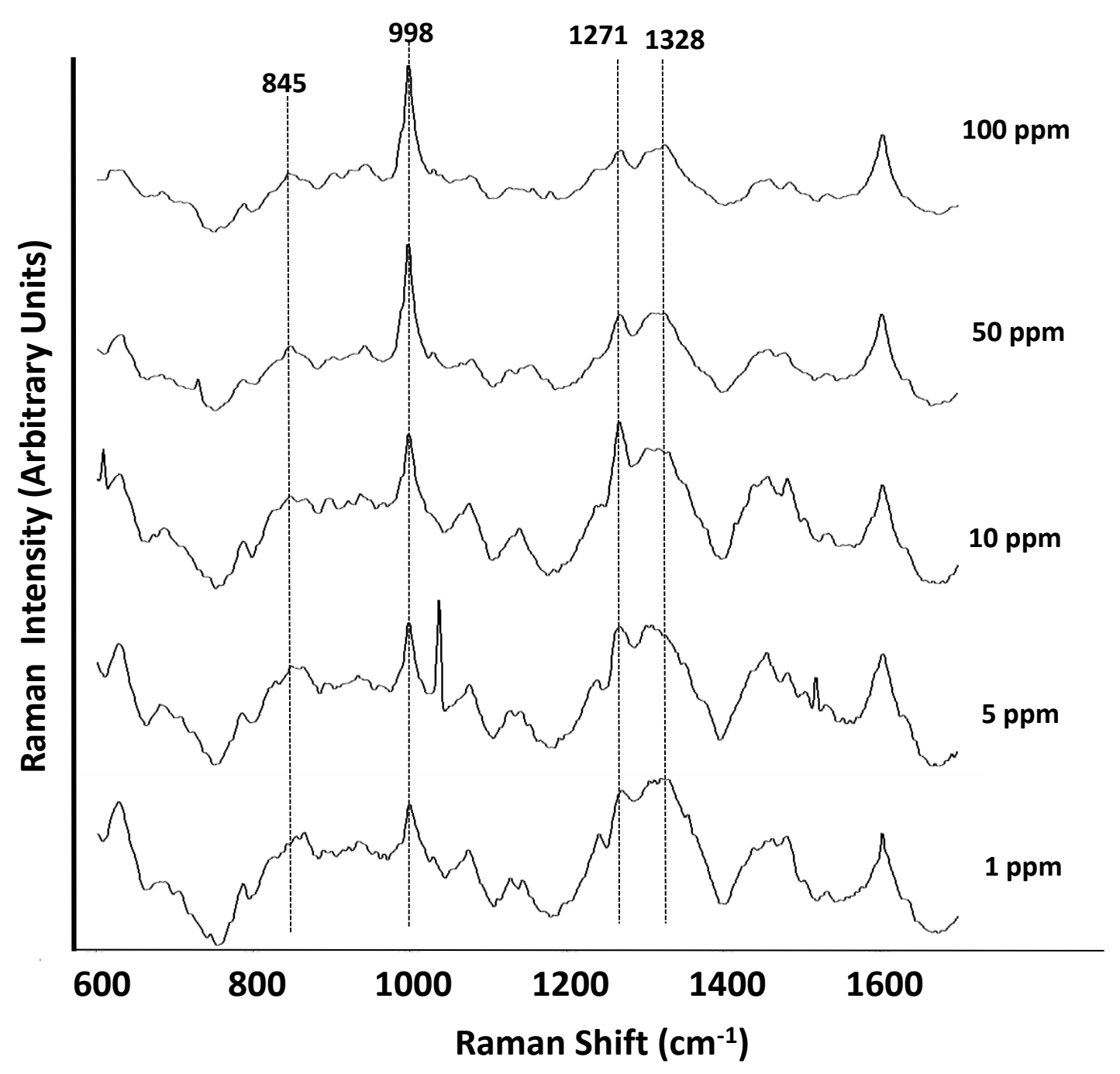

Figure 11. Average SERS spectra $(\mathrm{n}=7)$ of different concentrations of forchlorfenuron solutions extracted from grape skins. Spectra were processed by smoothing at $4 \mathrm{~cm}^{-1}$ and $2^{\text {nd }}$ order polynomial subtraction for baseline adjustment. 


\subsubsection{Second derivative transformation}

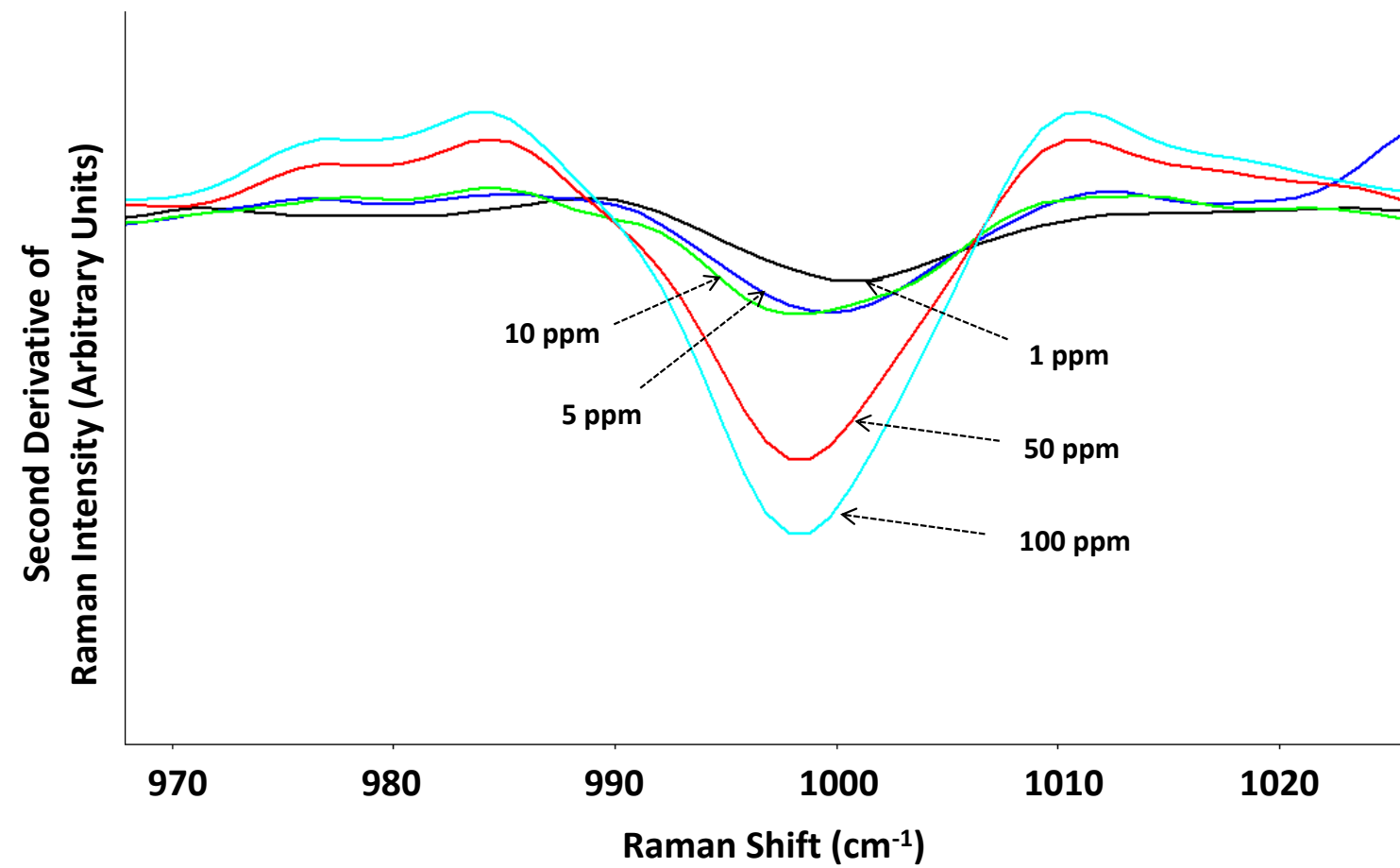

Figure 12. Second derivative transformation of average SERS spectra $(n=7)$ for different concentrations of forchlorfenuron solutions extracted from grape skins at $1000 \mathrm{~cm}^{-1}$.

The second derivative transformation of forchlorfenuron extraction shows the same pattern as the pure chemical with the most predominant peak at $998 \mathrm{~cm}^{-1}$. The signal intensities follow the same trend between different concentrations of forchlorfenuron ranging from 1 to $100 \mathrm{ppm}$.

\subsubsection{Partial least squares analysis (PLS)}

The lowest RMSEP value was achieved at six latent variables with R value of 0.94 and RMSEP of $1.305 \times 10^{-5}$ (Figure $\left.13 \& 14\right)$. 


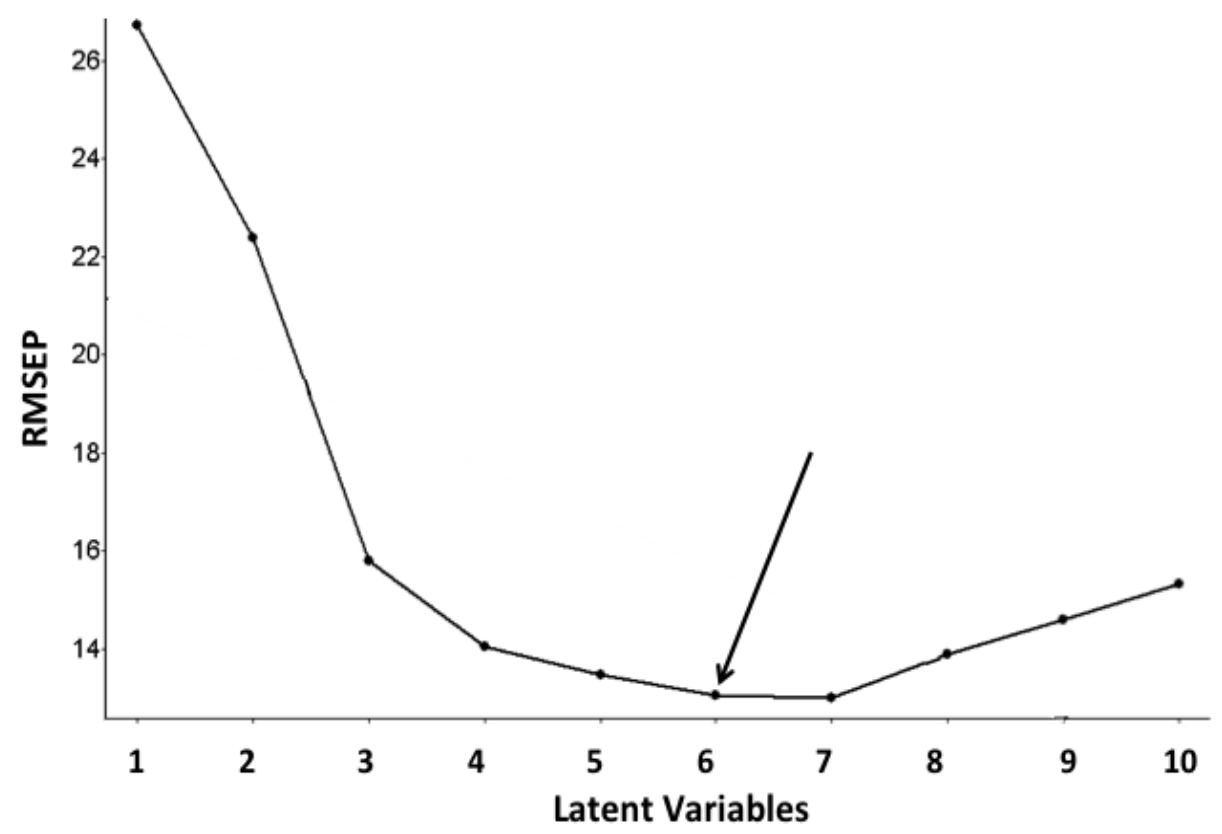

Figure 13. RMSEP values of the PLS models with different latent variables.

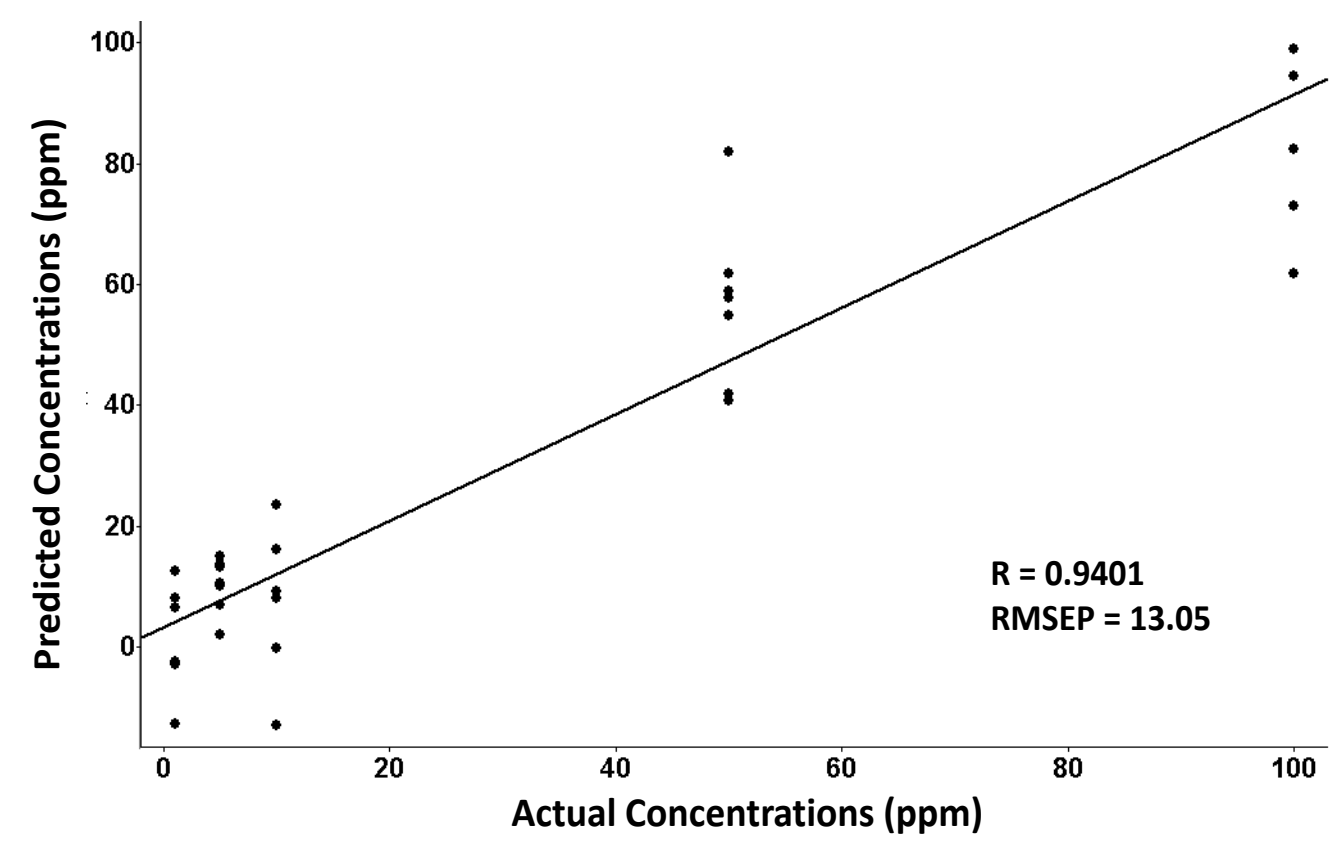

Figure 14. Predicted forchlorfenuron concentration ( $\mathrm{ppm}$ ) vs. actual puforchlorfenuron concentration (ppm) extracted from grape skin using PLS models at lowest latent variable. Data processed with smoothing at 4 $\mathrm{cm}^{-1}$, a $2^{\text {nd }}$ order polynomial subtraction for baseline adjustment, seven latent variables, spectral region from $600-1800 \mathrm{~cm}^{-1}$, spectral number $=35$. 


\subsubsection{Detection limit (DL)}

The DL of forchlorfenuron extracted from grape skin was calculated to be $4.43 \mathrm{ppm}$ (Table 2). This value is a little higher than that obtained from the pure sample, which is reasonable due to the additional extraction procedure.

\subsubsection{Recovery based on SERS method}

Previous studies suggest that the extracted samples should have the same composition as the standard pure samples (Sentellas and others 2001). Therefore, the recovery of forchlorfenuron extracted from grape skin was established in the same way as the pure chemical. Results show that the recoveries for pure chemical were $111.97 \%$ and $79.04 \%$ from concentrations at $50 \mathrm{ppm}$ and $100 \mathrm{ppm}$. For chemical extractions from grape skin the recovery were from 30 to $194.6 \%$ for concentrations ranging from 1 to $100 \mathrm{ppm}$ (Table 3). 
Table 3. Recovery of pure forchlorfenuron solutions

\begin{tabular}{lccc}
\hline \multicolumn{1}{c}{ Sample } & Spiked (ppm) & Quantified (ppm) & Recovery (\%) \\
\hline Pure Forchlorfenuron & 50 & 55.99 & 111.97 \\
& 100 & 79.04 & 79.04 \\
$\begin{array}{l}\text { Forchlorfenuron } \\
\text { extracted from grape } \\
\text { skin }\end{array}$ & 1 & 0.30 & 30.00 \\
& 5 & 9.73 & 194.60 \\
& 10 & 16.72 & 167.20 \\
& 50 & 57.06 & 114.12 \\
& 100 & 86.66 & 86.66 \\
\hline
\end{tabular}

\subsubsection{HPLC verification}

HPLC was used for further quantification of forchlorfenuron extracted from grape skin. A calibration curve was established by testing pure chemical solutions ranging from 0 to 100 ppm (Figure 15), exhibiting a linear regression with an $\mathrm{R}$ value of 0.99 . Five different concentrations of forchlorfenuron from 1 to $100 \mathrm{ppm}$ extracted from grape skin were then tested by HPLC and the recovery percentage was calculated based on the calibration curve, ranging from 72.2 to $158.4 \%$ accordingly (Table 4). The DL for forchlorfenuron by HPLC has been reported by previous studies to be $0.02 \mathrm{ppm}$ (Zhang and others 2010), which is lower than that of SERS. 
Table 4. Recovery of forchlorfenuron solutions extracted from grape skins based on HPLC calibration curve

\begin{tabular}{lllll}
\hline $\begin{array}{l}\text { Spiked } \\
\text { concentration } \\
(\mathrm{ppm})\end{array}$ & Area $\left(\mathrm{mAU}^{*} \mathrm{~s}\right)$ & Actual area & Quantified (ppm) Recovery $(\%)$ \\
\hline 1 & 26.034 & 260.34 & 1.58 & 158.40 \\
5 & 49.19 & 491.90 & 3.61 & 72.20 \\
10 & 94.69 & 946.90 & 7.59 & 75.90 \\
50 & 599.43 & 5994.30 & 51.78 & 103.60 \\
100 & 1116.01 & 11160.19 & 97.00 & 97.00 \\
\hline
\end{tabular}

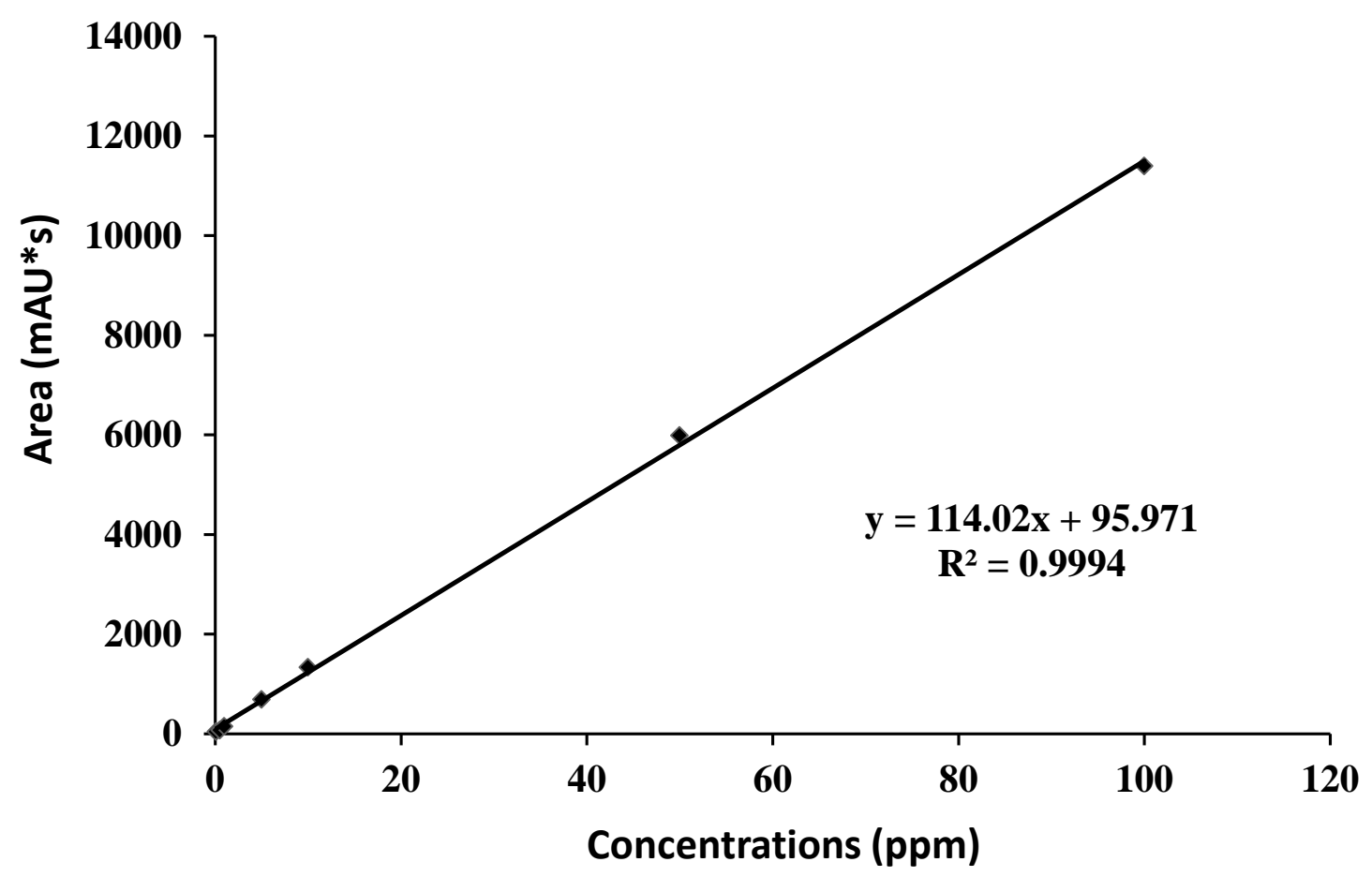

Figure 15. Calibration curve for forchlorfenuron measured by HPLC. 


\section{CHAPTER 5}

\section{CONCLUSIONS}

Forchlorfenuron extracted from grape skins was detected by SERS and HPLC methods. Characteristic SERS spectra of various concentrations of forchlorfenuron (0.1 to $100 \mathrm{ppm})$ were acquired using gold nanosubstrates. SERS is a rapid and reliable method for detection of chemical contaminants in various food matrices and there is a great potential in using gold-coated nanosubstrates in SERS. Results can be improved if better substrates and extraction protocols are used. SERS in combination with other methods such as HPLC will be a novel approach for rapid detection and discrimination of various chemical and biochemical contaminants in foods. A large number of food samples can be quickly screened by SERS, and the results can then be verified by HPLC. 


\section{REFERENCES}

Albrecht MG, Creighton JA. 1977. Anomalously intense Raman spectra of pyridine at a silver electrode.Jounal of the American Chemical Society 99(15):5215-7.

Alderman DJ. 1988. Fisheries chemotherapy: a review. Recent advances in aquaculture: Springer. p. 1-61.

Andersen WC, Turnipseed SB, Roybal JE. 2006. Quantitative and confirmatory analyses of malachite green and leucomalachite green residues in fish and shrimp. Journal of agricultural and food chemistry 54(13):4517-23.

Brown CA, Jeong K-S, Poppenga RH, Puschner B, Miller DM, Ellis AE, Kang K-I, Sum S, Cistola AM, Brown SA. 2007. Outbreaks of renal failure associated with melamine and cyanuric acid in dogs and cats in 2004 and 2007. Journal of Veterinary Diagnostic Investigation 19(5):525-31.

Cairns T, Sherma J. 1992. Emerging Strategies for Pesticides Analysis: CRC Press.

Campion A, Kambhampati P. 1998. Surface-enhanced Raman scattering. Chemical Society Reviews 27(4):241-50.

Chadha K, Shikhamany S. 1999. The grape: improvement, production and post-harvest management: Malhotra Publishing House.

Chan E, Griffiths SM, Chan C. 2008. Public-health risks of melamine in milk products. The Lancet 372(9648):1444-5.

Chen C, Qian Y, Chen Q, Tao C, Li C, Li Y. 2011. Evaluation of pesticide residues in fruits and vegetables from Xiamen, China. Food Control 22(7):1114-20.

Cialla D, März A, Böhme R, Theil F, Weber K, Schmitt M, Popp J. 2012. Surfaceenhanced Raman spectroscopy (SERS): progress and trends. Analytical and bioanalytical chemistry 403(1):27-54.

Cianciolo RE, Bischoff K, Ebel JG, Van Winkle TJ, Goldstein RE, Serfilippi LM. 2008. Clinicopathologic, histologic, and toxicologic findings in 70 cats inadvertently exposed to pet food contaminated with melamine and cyanuric acid. Journal of the American Veterinary Medical Association 233(5):729-37.

Culp SJ, Beland FA. 1996. Malachite green: a toxicological review. International Journal of Toxicology 15(3):219-38.

Davies PJ. 1995. Plant hormones: physiology, biochemistry and molecular biology: Kluwer Academic Dordrecht, The Netherlands. 
Dollish FR, Fateley WG, Bentley FF. 1974. Characteristic Raman frequencies of organic compounds: Wiley New York.

EPA. 2004. Pesticide fact sheet. Forchlorfenuron.

Fenik J, Tankiewicz M, Biziuk M. 2011. Properties and determination of pesticides in fruits and vegetables. TrAC Trends in Analytical Chemistry 30(6):814-26.

Fleischmann M, Hendra P, McQuillan A. 1974. Raman spectra of pyridine adsorbed at a silver electrode. Chemical Physics Letters 26(2):163-6.

Freeman SK, Lord RC. 1974. Applications of laser Raman spectroscopy. Physics Today 27:48.

Gremlich H-U, Yan B. 2001. Infrared and Raman spectroscopy of biological materials: CRC Press.

Han Z, Liu G, Rao Q, Bai B, Zhao Z, Liu H, Wu A. 2012. A liquid chromatography tandem mass spectrometry method for simultaneous determination of acid/alkaline phytohormones in grapes. Journal of Chromatography B 881:83-9.

Hau AK-c, Kwan TH, Li PK-t. 2009. Melamine toxicity and the kidney. Journal of the American Society of Nephrology 20(2):245-50.

Haynes CL, McFarland AD, Duyne RPV. 2005a. Surface-enhanced Raman spectroscopy. Analytical Chemistry 77(17):338 A-46 A.

Haynes CL, McFarland AD, Duyne RPV. 2005b. Surface-enhanced Raman spectroscopy. Analytical Chemistry 77(17):338 A-46 A.

Haynes CL, Van Duyne RP. 2001. Nanosphere lithography: a versatile nanofabrication tool for studies of size-dependent nanoparticle optics. The Journal of Physical Chemistry B 105(24):5599-611.

Hu J-Y, Li J-Z. 2006. Determination of forchlorfenuron residues in watermelon by solidphase extraction and high-performance liquid chromatography. Journal of AOAC International 89(6):1635-40.

Huang Y, Cavinato AG, Mayes DM, Bledsoe GE, Rasco BA. 2002. Nondestructive prediction of moisture and sodium chloride in cold smoked Atlantic salmon (Salmo salar). Journal of Food Science 67(7):2543-7.

Ingelfinger JR. 2008. Melamine and the global implications of food contamination. New England Journal of Medicine 359(26):2745-8. 
Jeanmaire DL, Van Duyne RP. 1977. Surface Raman spectroelectrochemistry: Part I. Heterocyclic, aromatic, and aliphatic amines adsorbed on the anodized silver electrode. Journal of Electroanalytical Chemistry and Interfacial Electrochemistry 84(1):1-20.

Jehlicka J, Culka A, Edwards HGM. 2010. Raman spectra of nitrogen-containing organic compounds obtained in high altitude sites using a portable spectrometer: Possible application for remote robotic Titan studies. Planetary and Space Science 58(5):875-81.

Jensen T, Kelly L, Lazarides A, Schatz GC. 1999. Electrodynamics of noble metal nanoparticles and nanoparticle clusters. Journal of Cluster Science 10(2):295-317.

Klots TD. 1995. Vibrational spectra of indene. Part 4. Calibration, assignment, and idealgas thermodynamics. Spectrochimica Acta Part A: Molecular and Biomolecular Spectroscopy 51(13):2307-24.

Klots TD, Collier WB. 1995. Heteroatom derivatives of indene Part 3. Vibrational spectra of benzoxazole, benzofuran, and indole. Spectrochimica Acta Part A: Molecular and Biomolecular Spectroscopy 51(8):1291-316.

Kneipp K, Kneipp H, Itzkan I, Dasari RR, Feld MS. 1999. Ultrasensitive chemical analysis by Raman spectroscopy. Chemical Reviews 99(10):2957-76.

Kneipp K, Kneipp H, Itzkan I, Dasari RR, Feld MS. 2002. Surface-enhanced Raman scattering and biophysics. J Phys Condens Matter 14(18):R597.

Le Ru E, Blackie E, Meyer M, Etchegoin P. 2007. Surface enhanced Raman scattering enhancement factors: a comprehensive study. The Journal of Physical Chemistry C 111(37):13794-803.

Le Ru E, Etchegoin P. 2008. Principles of Surface-Enhanced Raman Spectroscopy: and related plasmonic effects: Access Online via Elsevier.

Lee K, Irudayaraj J. 2009. Periodic and Dynamic 3-D Gold Nanoparticle- DNA Network Structures for Surface-Enhanced Raman Spectroscopy-Based Quantification. The Journal of Physical Chemistry C 113(15):5980-3.

Li-Chan ECY. 1996. The applications of Raman Spectroscopy in food science. Trends Food Science and Technology 7(11):361-70.

Lin M, He L, Awika J, Yang L, Ledoux DR, Li H, Mustapha A. 2008. Detection of melamine in gluten, chicken feed and processed foods using surface enhanced Raman spectroscopy and HPLC. Journal of Food Science 73(8):T129-T34. 
Litzau JJ, Mercer GE, Mulligan KJ. 2008. GC-MS screen for the presence of melamine, ammeline, ammelide, and cyanuric acid. US FDA Laboratory Information Bulletin 4423.

Liu B, Zhou P, Liu X, Sun X, Li H, Lin M. 2013a. Detection of pesticides in fruits by surface-enhanced raman spectroscopy coupled with gold nanostructures. Food and Bioprocess Technology 6(3):710-8.

Liu B, Zhou P, Liu X, Sun X, Li H, Lin M. 2013b. Detection of pesticides in fruits by surface-enhanced raman spectroscopy coupled with gold nanostructures. Food Bioprocess Technology 6(3):710-8.

Lu X, Al-Qadiri HM, Lin M, Rasco BA. 2011. Application of mid-infrared and Raman spectroscopy to the study of bacteria. Food Bioprocess Technology 4(6):919-35.

Mansour HM, Hickey AJ. 2007. Raman characterization and chemical imaging of biocolloidal self-assemblies, drug delivery systems, and pulmonary inhalation aerosols: a review. Journal of the American Association of Pharmaceutical Scientists: PharmSciTech 8(4):140-55.

McFarland AD, Young MA, Dieringer JA, Van Duyne RP. 2005. Wavelength-scanned surface-enhanced Raman excitation spectroscopy. The Journal of Physical Chemistry B 109(22):11279-85.

Moskovits M. 2005. Surface-enhanced Raman spectroscopy: a brief retrospective. Journal of Raman Spectroscopy 36(6-7):485-96.

Naumann D. 2000. Infrared spectroscopy in microbiology. In: Encyclopedia of analytical chemistry. p 102-131.

Park W-H, Kim ZH. 2010. Charge transfer enhancement in the SERS of a single molecule. Nano letters 10(10):4040-8.

Puschner B, Poppenga RH, Lowenstine LJ, Filigenzi MS, Pesavento PA. 2007. Assessment of melamine and cyanuric acid toxicity in cats. Journal of Veterinary Diagnostic Investigation 19(6):616-24.

Romo-Herrera JM, Alvarez-Puebla RA, Liz-Marzán LM. 2011. Controlled assembly of plasmonic colloidal nanoparticle clusters. Nanoscale 3(4):1304-15.

Schatz G. 2001. Electrodynamics of nonspherical noble metal nanoparticles and nanoparticle aggregates. Journal of Molecular Structure: THEOCHEM 573(1):7380. 
Schatz GC, Van Duyne RP. 2002. Electromagnetic Mechanism of Surface-Enhanced Spectroscopy. In: J. M. Chalmers and P. R. Griffiths, Editor. Handbook of vibrational spectroscopy. New York: Wiley. p $759-774$.

Sentellas S, Saurina J, Hernández-Cassou S, Galceran MT, Puignou Ls. 2001. Multivariate calibration methods for quantification in strongly overlapping capillary electrophoretic peaks. Journal of Chromatography A 909(2):259-69.

Sharma D, Awasthi MD. 2003. Behaviour of forchlorfenuron residues in grape, soil and water. Chemosphere 50(5):589-94.

Smith E, Dent G. 2005. Modern Raman spectroscopy: a practical approach. London, Wiley. $72 \mathrm{p}$.

Smoker M, Krynitsky AJ. 2008. Interim method for determination of melamine and cyanuric acid residues in foods using LC-MS/MS: version 1.0. US FDA Laboratory Information Bulletin (4422).

Suárez-Pantaleón C, Wichers J, Abad-Somovilla A, van Amerongen A, Abad-Fuentes A. 2012. Development of an immunochromatographic assay based on carbon nanoparticles for the determination of the phytoregulator forchlorfenuron. Biosensors and Bioelectronics. 42: 170-176.

Tu Q, Chang C. 2012. Diagnostic applications of Raman spectroscopy. Nanomedicine: Nanotechnology, Biology and Medicine 8(5):545-58.

Turnipseed SB, Walker CC, Roybal JE, Pfenning AP, Hurlbut JA. 1998. Confirmation of fluoroquinolones in catfish muscle by electrospray liquid chromatography/mass spectrometry. Journal of AOAC International 81(3):554.

Valverde A, Aguilera A, Ferrer C, Camacho F, Cammarano A. 2010. Analysis of forchlorfenuron in vegetables by LC/TOF-MS after extraction with the buffered QuEChERS method. Journal of Agricultural and Food Chemistry 58(5):2818-23.

Van Duyne RP. 1979. Laser excitation of Raman scattering from adsorbed molecules on electrode surfaces. Chemical and Biochemical Applications of Lasers 4:101.

Willets KA, Van Duyne RP. 2007. Localized surface plasmon resonance spectroscopy and sensing. Annual Review of Physical Chemistry 58:267-97.

Xin H, Stone R. 2008. Chinese probe unmasks high-tech adulteration with melamine. Science 322(5906):1310-1.

Zhai F, Huang Y, Li C, Wang X, Lai K. 2011. Rapid determination of ractopamine in swine urine using surface-enhanced Raman spectroscopy. Journal of Agricultural and Food Chemistry 59(18):10023-7. 
Zhang H, Yang X, Dong AJ, Ma Y, Wang ZY, Wang J, Yang L, Zhang YC. 2010. Residue analysis of forchlorfenuron in fruit and vegetable by RP-HPLC.

Bioinformatics and Biomedical Engineering (iCBBE), 2010 4th International Conference on: IEEE. p. 1-4. 


\section{VITA}

Haobing Qian was born on November $11^{\text {th }}, 1989$ in Wuxi, Jiangsu, China. She graduated from Shanghai Kongjiang Senior High School in July 2007 and went to Jiangnan University in Wuxi to study Food Science and Technology. In July 2011, she obtained Bachelor of Science degree in Food Science and Technology from Jiangnan University. She joined the Department of Food Science at the University of Missouri in Columbia, Missouri in July 2011 to pursue her Master's degree in Food Science. 